\title{
An Undominated Mechanism for a Class of Informed Principal Problems with Common Values*
}

\author{
Dieter Balkenborg $^{\dagger} \quad$ Miltiadis Makris ${ }^{\ddagger}$
}

25th February 2015

\begin{abstract}
In a class of informed principal problems with common values, we define iteratively a particular allocation which we call the assured allocation. It is comparatively easy to calculate and straightforward to interpret. It always exists, is unique and continuous in the priors. It is undominated, i.e. efficient among the different types of the principal subject to the agent's interim participation constraint. It is a perfect Bayesian equilibrium of the three-stage game in Myerson (1983) and Maskin and Tirole (1992). It dominates the RSW allocation as defined in Maskin and Tirole (1992) and coincides with it when the latter is undominated. It is the unique neutral optimum as defined in Myerson (1983) when there are only two types. When the assured allocation is separating, then it is a neutral optimum with three or more types. It is an equilibrium of a game of competition in a market with adverse selecton.
\end{abstract}

Keywords: Mechanism design, informed principal, common values, neutral optimum.

JEL classification: D82, D86

\footnotetext{
${ }^{*}$ We would like to thank the Editor and two anonymous referees for very helpful comments and suggestions that have significantly improved the paper. We would also like to thank Theodoros Diasakos, Christopher Hennesy, Kostas Koufopoulos, Roger Myerson, Tymofiy Mylovanov, Ludovic Renou, Jean Tirole, Yiannis Vailakis and participants at seminars at the Institute for Advanced Studies in Vienna and the Universities of Maastricht, Göttingen and Bielefeld for comments and helpful discussions prior to this draft. The usual disclaimer applies.

${ }^{\dagger}$ Department of Economics, University of Exeter, Rennes Drive, Streatham Court, Exeter, EX, UK. E-mail: D.G.Balkenborg@exeter.ac.uk

$\ddagger$ Corresponding author; Department of Economics, University of Southampton, University Rd., Southampton, SO17 1BJ, UK. E-mail: M.Makris@soton.ac.uk.
} 


\section{Introduction}

Informed principal problems are adverse selection problems where the principal, who proposes a contract to an agent, has private information. Important examples are firms with private information about projects who seek finance from competing lenders, or managers with private information who have bargaining power when dealing with shareholders. Informed principal problems may also be important for the analysis of regulated privatization when the government has better information about the productivity of its asset, or for the analysis of regulation of a monopolistic FDI when the government has superior information about the characteristics of the domestic market.

Different types of the informed principal may offer different contracts, and hence the choice of a contract itself becomes a signal to the agent regarding the principal's type. This gives rise to an inference process that affects the agent's incentives and willingness to accept the contract. Consequently, informed principal problems are significantly harder to understand than models where only the agent has private information. This difficulty arises in particular in the case of the so-called common value problems, where typically the optimum for the principal under complete information is not incentive compatible when the principal has superior information. ${ }^{1}$ In this paper we are primarily interested in the less studied case of common values, ${ }^{2}$ and we restrict attention to a natural extension of the canonical adverse selection model that allows for common values. For a discussion of the relevance of common value problems, we refer the reader to the excellent discussion in Maskin and Tirole (1992).

Different ways of resolving the conflict between the principal's types regarding the contract offer has given rise to different solution concepts. For our framework, the two main solutions are the neutral optimum and the RSW (Rothschild-Stiglitz-Wilson) allocation. The neutral optimum, introduced by Myerson (1983), is a strong refinement motivated by

\footnotetext{
${ }^{1}$ In the case of private values, i.e. when the principal's private information does not directly affect the agent's utility, it is typically possible to find, at least when utilities are quasi-linear, mechanisms that (a) are efficient among the different types of the principal when the agent's beliefs coincide with her priors, and (b) give the agent an expected payoff at least as high as his outside option, even if the agent knew the type of the principal,. For the case of private values we refer to Maskin and Tirole (1990), and the recent works of Cella (2008) and Mylovanov and Tröger (2012) together with the literature they cite.

${ }^{2}$ A common values environment has also been studied in Maskin and Tirole (1992) and Severinov (2009). The results in the latter work do not apply here, because we focus on problems with one agent, which is ruled out by assumption in Severinov (2009).
} 
various cooperative and non-cooperative solution concepts, which makes it a compelling solution concept. The RSW allocation, studied by Maskin and Tirole (1992) in terms of informed principal problems, is the "least-costly-allocation" that has been emphasized in the signaling and screening literature because it is prior-free (i.e. it only depends on the support of the prior) and survives the intuitive criterion of Cho and Kreps (1987). Its importance for informed principal problems is that, if offered, it will be (weakly) preferred over the outside option by the agent regardless of his/her beliefs.

This paper contributes to this literature by introducing a novel solution concept, the assured allocation, which is shown to possess a number of attractive properties. The assured allocation exists, is unique and, in contrast to the RSW, is continuous in the priors of the agent, even if the probabilities of some types go to zero. Moreover, it can easily be interpreted. Namely, the assured allocation guarantees for each possible type the surplus he could have attained, had there been no other types with higher productivity, conditional on the same being true for all types of lower productivity. ${ }^{3}$ The assured allocation is an undominated mechanism, i.e. efficient among the different types of the principal when the agent's interim participation constraint must be satisfied, amongst all deterministic mechanisms. If there is no bunching in the assured allocation, then we can show that the assured allocation is undominated also amongst all randomizing mechanisms.

The assured allocation weakly dominates the RSW allocation. It coincides with the RSW allocation when the latter is undominated. A similarity between the assured allocation and the RSW is that both can be calculated recursively via a sequence of optimization problems for the different types.

We know from Maskin and Tirole (1992) that an equilibrium selection issue arises in their mechanism-selection game when the RSW allocation is dominated: all allocations that weakly dominate it are perfect Bayesian equilibria, and so the assured allocation is always such an equilibrium. ${ }^{4}$ When there are only two types we can show that the assured allocation coincides with the core mechanism as defined in Myerson (1983). This provides a very strong equilibrium selection argument in favor of the assured allocation,

\footnotetext{
${ }^{3}$ The assured allocation can be understood as a solution to a principal-agent problem where the outside option varies with the type, as in Jullien (2000). In our case the outside options are, however, defined endogenously.

${ }^{4}$ For related work see Ma (1994) and von Thadden (1995).
} 
and emphasizes the importance of the assured allocation for the case of two types.

However, the above result cannot be extended to the case of more than two types. Therefore, one might ask whether there exists a game of which the assured allocation is the unique equilibrium outcome. This is a very hard question to tackle in its full generality in informed principal problems with common values. Nevertheless, we can show that there is an extensive form game for which the assured allocation can be an equilibrium, when the RSW is not. This game draws on the insurance literature. It has competing uninformed parties simultaneously posting contracts and subsequently deciding whether to withdraw their contracts, before the informed party chooses, given available contracts, who to trade with and under what terms. ${ }^{5}$ We know from the insurance literature (e.g. Rothschild and Stiglitz (1976)) that the RSW allocation will not survive such a competitive process if high types in our set up (low risk types in the insurance model) are very likely: a profitable pooling contract that dominates the RSW allocation will be introduced attracting thus all high types. In this paper, we show, on the other hand, that the assured allocation will survive such a competitive process if priors are also such that the agent makes profits only from the highest type under the assured allocation. Crucially, this set of priors is non-empty. This finding is a generalization of related results in Myazaki (1977) and Spence (1978) who study Wilson's "anticipatory equilibria" (Wilson (1977)): the former does so in the context of a labor market with two types that fits our framework, while the latter does so in a multi-type insurance model that does not fit our set up. ${ }^{6}$ This weak implementation result adds, we believe, significantly to the value of the assured allocation. It provides, alongside the fact that the assured allocation dominates the RSW allocation, an equilibrium selection argument in favour of the assured vis-a-vis the RSW allocation when there are more than two types. This equilibrium selection argument is reinforced by the fact that the assured allocation is shown to be a neutral optimum under conditions that are similar to those in Jullien (2000) and imply that there is no bunching in the assured allocation. Given our aforementioned weak implementation result, the assured allocation might also be useful in understanding outcomes in competitive markets with adverse selection.

\footnotetext{
${ }^{5}$ A similar game is investigated in Hellwig (1987).

${ }^{6}$ See also the more recent study of a two-type insurance model in Diasakos and Koufopoulos (2009).
} 
Neutral optima are in general very difficult to calculate in practice, which is possibly one reason why there are very few applications using this solution concept. ${ }^{7}$ Uniqueness of the neutral optimum is also not generally known. We regard it hence as a major contribution of this paper that we can show that the assured allocation is a neutral optimum under conditions ruling out bunching, and that neutral optimum and assured allocation coincide when there are only two types. ${ }^{8}$ However, neutral optimum and the assured allocation do not coincide in general. In fact, we provide an example of three types where the assured allocation involves bunching between the various types and is dominated by a randomized mechanism, and hence is not a neutral optimum.

The organization of the paper is as follows. In Section 2 we describe the model and the weakest assumptions for which we know our analysis to hold. Section 3 introduces the assured allocation and derives some basic properties. Section 4 discusses some examples of the assured allocation, and it demonstrates a case when the assured allocation involves bunching and is not a neutral optimum. Section 5 reviews a number of central concepts in the literature, and compares the assured allocation with the RSW allocation and the neutral optimum. Section 6 discusses a particular extensive-form game for which the assured allocation is, in contrast to the RSW allocation, always an equilibrium. Section 7 concludes. Proofs omitted from the main text are given in the appendix.

\section{The Model}

We will be focusing on a model of a producer/seller and a buyer, in a broad sense, where the producer's cost-efficiency/productivity "type" is her private information. We assume quasi-linear preferences and a finite type set, ${ }^{9}$ and that all types of the producer have the

\footnotetext{
${ }^{7}$ The only applications we know of are the discussion of the lemon problem in Myerson (1985) and the extended liability problem discussed in Balkenborg (2006), which are both common value problems. Both problems do not fall into our framework. Applications to bargaining problems with two-sided incomplete information are also given in Myerson (1984) and Darrough and Stoughton (1989).

${ }^{8}$ Other papers characterizing neutral optima are Mylovanov and Tröger (2012), Cella (2008) and Severinov (2009). Mylovanov and Tröger (2014) and Cella (2008) do this in the private value context.

${ }^{9}$ See also Severinov (2009) and Mylovanov and Tröger (2012). A finite type set is also used by Myerson (1983) and Maskin and Tirole (1992), and so we adopt the same assumption to facilitate comparisons. Note, however, that the general framework of Maskin and Tirole (1992) does not require quasi-linearity.
} 
same outside option. ${ }^{10}$ We also allow for common values: the buyer's utility depends on the seller's productivity type. The model we focus on is the canonical model of adverse selection used, for instance, in the textbook Laffont and Martimort (2002), Chapters 2 and 3.1, except that we allow for common values and assume that the informed seller is the side with full bargaining power. As we will explain at the end of this section, this model differs qualitatively from the standard insurance model; e.g. Stiglitz (1977) and Spence (1978). The model we work with fits the example in Maskin and Tirole (1992) of an uninformed firm owner and an informed manager who interact to determine the latter's output and compensation.

The seller (the principal) produces $q \geq 0$ units of a product at a total cost which depends on output and the seller's cost-efficiency type. Let the finite type set be, without loss of generality, the set of numbers $\mathcal{N}=\{1,2, \ldots, N\}$. The production cost of $q$ units by type $i \in \mathcal{N}$ is denoted by $C_{i}(q)$, which is a twice continuously differentiable function. We denote the prior probability of type $i, 1 \leq i \leq N$, by $s_{i}$. In the following, let $f_{i} \equiv \sum_{j=1}^{i} s_{j}$, with the convention that $f_{0}=0$. The type of the seller is his private knowledge and the distribution of types is common knowledge.

Costs and marginal costs are non-decreasing in $q$, i.e.

$$
C_{i}^{\prime} \geq 0, C_{i}^{\prime \prime} \geq 0
$$

for all $i \in \mathcal{N}$ and $q \geq 0$. They are also decreasing in the type, i.e. if $i<j$ then

$$
\begin{aligned}
& C_{j}(q)<C_{i}(q) \text { for } q>0 \\
& C_{j}^{\prime}(q)<C_{i}^{\prime}(q) \text { for } q \geq 0
\end{aligned}
$$

The latter is a sorting condition that ranks types according to their marginal utility from trade. It states that higher (i.e. more productive) types value trading with the agent more.

The value of the product of type- $i$ seller to the buyer (the agent) is $S_{i}(q)$, which is a twice continuously differentiable function. The value of the product is non-decreasing and

\footnotetext{
${ }^{10}$ Therefore, our model is not suitable to investigate the problem of insurance (see Stiglitz (1977) and Maskin and Tirole (1992)) or of franchising (see Maskin and Tirole (1992)).
} 
the marginal value non-increasing in the level of output:

$$
S_{i}^{\prime} \geq 0 \quad S_{i}^{\prime \prime} \leq 0
$$

for all $i \in \mathcal{N}$ and $q \geq 0$. In the case of independent values, $S_{i}(q)$ is independent of $i$. As we are primarily interested in the case of common values, we assume that the value of the product is non-decreasing in seller's type. Thus, if $i<j$ then

$$
S_{j}(q) \geq S_{i}(q) \text { for } q \geq 0
$$

This assumption simply says that higher types (who have lower costs) are more productive. It is used in the proofs of all our theorems, except Theorem 2.

The net surplus is concave

$$
S_{i}^{\prime \prime}<C_{i}^{\prime \prime}
$$

In addition, we assume that:

$$
\lim _{q \rightarrow \infty}\left(S_{N}(q)-C_{N}(q)\right)=-\infty
$$

which ensures that optimal output levels are always finite. Fixed costs are not too high:

$$
S_{1}(0) \geq C_{1}(0)
$$

We also assume that there are gains from trade (allowed to be zero for the lowest type), which are increasing in the seller's type:

$$
\begin{gathered}
S_{i}^{\prime}(0) \geq C_{i}^{\prime}(0) \\
S_{j}^{\prime}(q)-C_{j}^{\prime}(q)>S_{i}^{\prime}(q)-C_{i}^{\prime}(q) \text { for } i<j, \quad q \geq 0 .
\end{gathered}
$$

These conditions imply that the participation constraints for the various types of the principal are never binding in the allocations defined and discussed below. ${ }^{11}$ We therefore

\footnotetext{
${ }^{11}$ This follows from the fact that the reservation payoff of the principal will be assumed to be zero, and in the allocations defined below high types will not want to mimic lower types and the lowest type will get
} 
ignore them in what follows.

To reduce notation clustering, throughout we will use the following notations:

$$
\begin{aligned}
W_{i}(q) & :=S_{i}(q)-C_{i}(q) \text { for } 1 \leq i \leq N \\
\psi_{i}(q) & :=C_{i}(q)-C_{i+1}(q) \text { for } 1 \leq i<N
\end{aligned}
$$

Thus, $W_{i}(q)$ is the surplus generated from trade between the buyer and a seller of type $i$ that involves output $q$. Moreover, $\psi_{i}(q)$ is the cost-gain in the production of $q$ by having a seller of type $i+1$ instead of type $i$. Alternatively, it represents the cost disadvantage the seller of type $i$ would have when mimicking the more productive type $i+1$.

Next, we introduce some more assumptions and discuss the implications of all our assumptions. So far our assumptions imply that the type-dependent net surplus $W_{i}$ is a concave function, and that the full information production level $q_{i}^{o}$ exists, is unique and non-negative for $i=1$ and increasing in type $i$. In addition, $W_{1}\left(q_{1}^{o}\right) \geq 0$, and $W_{i}\left(q_{i}^{o}\right)$ is increasing in type $i$. Moreover, each $\psi_{i}(q)$ is positive for $q>0$ and an increasing function of $q$. We will also assume that it is (weakly) concave. That is,

Assumption A $\psi_{i}^{\prime \prime}(q) \leq 0$ for all $1 \leq i<N$ and $q \geq 0$.

We need Assumption $\mathrm{A}$ in order to guarantee that the problem $X_{n}$, discussed shortly when we introduce the assured allocation, is convex for every $n$.

The principal and the agent are involved in the following three-stage mechanismselection game $\Gamma$ : First the informed principal offers a mechanism to the agent in a takeor-leave-it manner. The aim of the mechanism is to fix the output $q$ to be produced by the seller and the transfer $t$ the buyer has to pay in return to the seller. A mechanism is a set of announcements for the principal and a rule that maps announcements to (possibly lotteries over) transfer-output pairs. ${ }^{12}$ The agent must then accept or reject the offered mechanism. If the agent accepts the mechanism then the latter is executed: the principal chooses her announcement and the associated transfer-output pair is implemented. We at least the first-best surplus. These will be ensuring a positive payoff due to the conditions in question.

${ }^{12}$ Because the agent has no private information, there is no need to include announcements of the agent into the mechanism. 
assume that the principal's and agent's reservation payoffs are zero. ${ }^{13}$

Most of the work in this literature (e.g. Myerson (1983), Maskin and Tirole (1992), Severinov (2009) and Mylovanov and Tröger (2012), Mylovanov and Tröger (2014)) use the same extensive form game in their study of the informed principal problem (adjusting for the number of agents and their information). We will restrict attention to perfect Bayesian equilibria (for a formal definition, see page 8 of Maskin and Tirole (1992)) of the three-stage game $\Gamma$. For brevity, we will sometimes simply speak of "equilibria" in what follows.

Moreover, applying the inscrutability principle in Myerson (1983), Section 3, we restrict without loss of generality attention to equilibria of the mechanism-selection game $\Gamma$ where all types of the principal propose the same mechanism. The proposal (first-) stage of game $\Gamma$ is hence uninformative and does not change the prior. Notice thus that there is pooling at the proposal stage, while there can be separation in the mechanism itself in the sense that different types of the principal can produce different quantities and receive different transfers. Following Maskin and Tirole (1992) we focus on finite simultaneous-actions mechanisms. By the revelation principle for Bayesian games, we have, for any mechanism offered by the principal and for given beliefs after the mechanism has been offered, that any equilibrium of the mechanism corresponds to a truthful equilibrium of a direct revelation mechanism (DRM). In such an equilibrium, the principal simply announces truthfully her type and the corresponding (stochastic) transfer-output pair is implemented. This resulting allocation, i.e. profile of (stochastic) transfer-output pairs per type, is the same with the one arising from the equilibrium of the general mechanism. We therefore focus on DRMs. Note that an allocation is effectively a DRM.

\subsection{Preliminaries}

Let a stochastic DRM be denoted with $\kappa \equiv\left(\kappa_{i}\right)_{1 \leq i \leq N} \equiv\left(\left(t_{i}, \mu_{i}(q)\right)\right)_{1 \leq i \leq N} \cdot \mu_{i}(q)$ denotes a probability measure over the set of feasible outputs $q \geq 0$ and describes a randomization over possible output levels designed for type $i$ as part of the stochastic DRM. In addition, $t_{i}$

\footnotetext{
${ }^{13}$ In the general model of Maskin and Tirole (1992) the reservation utility of the buyer is allowed to be dependent of the seller's type.
} 
denotes the net ${ }^{14}$ transfer from the buyer to the seller of type $i$. A stochastic mechanism can in general include a randomization over transfers as well. Due to quasi-linearity of utilities we can however restrict attention without loss of generality to stochastic mechanisms with deterministic transfers $\left(t_{i}\right)_{1 \leq i \leq N}$ (one would only have to replace a randomized transfer with its corresponding expected transfer).

Because we restrict attention to equilibria where the proposal stage is uninformative we can write without loss of generality the agent's participation constraint along the equilibrium in terms of the prior: ${ }^{15}$

$$
\sum_{i=1}^{N} s_{i}\left(\int S_{i}(q) d \mu_{i}(q)-t_{i}\right) \geq 0
$$

We will refer to a mechanism that satisfies the participation constraint as an individuallyrational mechanism. An ex post individually-rational mechanism satisfies $\int S_{i}(q) d \mu_{i}(q)-$ $t_{i} \geq 0$ for all $1 \leq i \leq N$.

Moreover, an allocation $\kappa$ satisfies incentive compatibility if the following incentivecompatibility constraints hold:

$$
t_{i}-\int C_{i}(q) d \mu_{i}(q) \geq t_{j}-\int C_{i}(q) d \mu_{j}(q) \text { for all } 1 \leq i, j \leq N
$$

This simply says that type $i$ prefers option $\kappa_{i}$ to any other option $\kappa_{j}, j \neq i$.

We now introduce some essential definitions. First,

Definition 1 A mechanism is feasible if it is incentive-compatible and individuallyrational.

More generally we will say that a mechanism is feasible given a non-empty subset of types $I \subseteq\{1, \ldots N\}$ if it is feasible conditional on the agent/buyer holding posterior beliefs that all possible types belong to $I$.

We next have:

\footnotetext{
${ }^{14}$ Though we allow for net transfers to be negative in the optimal allocation all transfers will be positive.

${ }^{15}$ Notice, however, that this simplification would be wrong for contracts offered off the equilibrium path. For our purpose this simplification is without loss of generality because we will not have to consider off equilibrium mechanisms.
} 
Definition 2 A mechanism is undominated if it is feasible and there does not exist another feasible mechanism which gives every type of the principal at least the same utility and some of his types a strictly higher utility.

Undominated mechanisms are important because if the principal can effectively communicate with the agent, then he should be expected to offer only undominated mechanisms (see page 1775 of Myerson (1983) for more on this).

A strengthening of the notion of undominated mechanisms is the concept of core mechanisms, also proposed by Myerson (1983). To introduce this concept start with a mechanism $\mu$, and consider the alternative mechanism $\nu$. Let $w$ be the set of winner types in $\nu$ compared to $\mu$. Mechanism $\nu$ blocks $\mu$ if it is feasible ("incentive compatible" in Myerson (1983) jargon) given any superset of $w$. We then have:

Definition 3 A mechanism $\mu$ is a core mechanism if there is no alternative mechanism that can block mechanism $\mu$.

Unless otherwise stated (e.g. Theorem 2), we restrict attention to deterministic mechanisms in this paper. Such a DRM is thus an option contract $\kappa \equiv\left(\kappa_{i}\right)_{1 \leq i \leq N} \equiv$ $\left(\left(t_{i}, q_{i}\right)\right)_{1 \leq i \leq N}$, where $q_{i}$ denotes the quantity produced and sold by the seller of type $i{ }^{16}$ For expositional simplicity, let us refer, hereafter, with some abuse of terminology, to a deterministic DRM as, simply, a mechanism or contract or allocation.

Denote the payoff of the principal of type $i$ under some mechanism $\kappa$ by $U_{i}=t_{i}-C_{i}\left(q_{i}\right)$. From now on we will, unless stated otherwise, use the substitution $U_{i}=t_{i}-C_{i}\left(q_{i}\right)$ and describe a contract as an $N$-tuple $\left(\left(U_{i}, q_{i}\right)\right)_{1 \leq i \leq N} \equiv\left(m_{i}\right)_{1 \leq i \leq N} \equiv m$. Under such reparametarization, the participation constraint can be re-written as

$$
\sum_{i=1}^{N} s_{i}\left(W_{i}\left(q_{i}\right)-U_{i}\right) \geq 0
$$

This states that the expected surplus must exceed the expected net payments to the seller.

One can also show, following standard steps (see the Appendix), that:

\footnotetext{
${ }^{16}$ As usual, for notatiional simplicity, we identify the Dirac measure over output level $q_{i}$ with $q_{i}$ itself.
} 
Lemma 1 For a mechanism to be incentive-compatible, it is necessary and sufficient that

$$
\begin{gathered}
U_{i} \geq U_{i+1}-\psi_{i}\left(q_{i+1}\right) \text { for } 1 \leq i<N \\
U_{i+1} \geq U_{i}+\psi_{i}\left(q_{i}\right) \text { for } 1 \leq i<N .
\end{gathered}
$$

This Lemma ensures that, when investigating for incentive-compatible allocations, we can restrict attention to the above constraints. We will be referring to (2) as the (local) upward incentive (-compatibility) constraint for type $i$, and to (3) as the (local) downward incentive (-compatibility) constraint for type $i+1$. The former simply says that type $i$ must receive as much utility as type $i+1$ minus the cost disadvantage he would have when imitating the more productive type $i+1$. The latter incentive constraint has a similar interpretation.

Remark 1 The above two local incentive-compatibility constraints imply that

$$
q_{i} \leq q_{i+1} \text { for } 1 \leq i<N .
$$

This follows directly by summing up the above two incentive constraints and using the monotonicity properties of $\psi_{i}(q)$. We will also be referring to (4) as the monotonicity constraint for $i$.

Let us consider next the benchmark case of full information where the type of the principal is common knowledge. In this case, the incentive-compatibility constraints are irrelevant. The full-information monopoly contract, denoted by $m^{M}$, is thus given by

$$
\left.m^{M}=\left(U_{i}^{M}, q_{i}^{M}\right)_{1 \leq i \leq N}=\left(W_{1}\left(q_{i}^{o}\right), q_{i}^{o}\right)\right)_{1 \leq i \leq N}
$$

where $q_{i}^{o} \equiv \arg \max _{q \geq 0} W_{i}(q)$. Notice that the full-information monopoly contract is first best in the sense that it maximizes social surplus, and that each type of the principal receives all the surplus he generates. Note also that $q_{1}^{o} \leq \ldots \leq q_{N}^{o}$.

We leave this section by highlighting the main difference between our buyer/seller model and the standard insurance model; e.g. Stiglitz (1977) and Spence (1978). Suppose that the informed party faces the full-information monopoly contract, $m^{M}$, and this con- 
tract is not incentive compatible. Then, in our model, the informed party has an incentive to mimic a type who is associated with higher first-best full-information output. In the insurance model, on the other hand, (with higher types being those of lower risk), the informed party has an incentive to mimic a type who is associated with lower first-best full-information output/cover.

\section{The Assured Allocation}

This work introduces the assured allocation. As we will show, it is an equilibrium of the three-stage-game $\Gamma$. Our definition is based on a sequence of constrained optimization problems to be solved inductively. In this sense the computation is straightforward, at least in comparison to finding a neutral optimum. The main properties of this allocation and hence its importance for informed-principal problems with common values, and its relationship with the other known equilibrium allocations will be discussed in Sections 4-6.

To define the assured allocation, let $1 \leq n \leq N$. Suppose the numbers $V_{1}, \cdots, V_{n-1}$ have been defined. We define a mechanism $\left(U_{i}^{n}, q_{i}^{n}\right)_{1 \leq i \leq n}$ as the solution to, and the scalar $V_{n}$ as the maximal value of, the following constrained optimization problem referred to as $X_{n}:$

$$
V_{n} \equiv \max _{\left(U_{i}, q_{i}\right)_{1 \leq i \leq n}} U_{n}
$$

subject to

$$
\begin{gathered}
U_{i} \geq U_{i+1}-\psi_{i}\left(q_{i+1}\right) \quad \text { for } 1 \leq i \leq n-1 \\
q_{i+1} \geq q_{i} \quad \text { for } 1 \leq i \leq n-1 \\
U_{i} \geq V_{i} \quad \text { for } 1 \leq i \leq n-1 \\
\sum_{i=1}^{n} s_{i}\left(W_{i}\left(q_{i}\right)-U_{i}\right) \geq 0
\end{gathered}
$$

We call $\left(U_{i}^{n}, q_{i}^{n}\right)_{1 \leq i \leq n}$ the $n$-assured allocation. ${ }^{17}$ For $n=N$ we speak for short of the

\footnotetext{
${ }^{17}$ Note that $(\mathrm{PC})$ can be rewritten as$$
\sum_{i=1}^{n} s_{i}^{n}\left(W_{i}\left(q_{i}\right)-U_{i}\right) \geq 0
$$

where $s_{i}^{n}=\frac{s_{i}}{s_{1}+\cdots+s_{n}}$. 
assured allocation. We call $V_{n}$ the assured claim of type $n$ and use it in the definition of problem $X_{j}, n+1 \leq j \leq N$. $^{18}$

We call the constraints $U_{i} \geq V_{i}$ the assured claim constraints. The rest of the constraints are, respectively, the incentive-compatibility constraints that require that every type $i$ does not have an incentive to mimic type $i+1,1 \leq i<N-1$, the monotonicity constraints that require output is non-decreasing, and the participation constraint.

We can regard the assured allocation as a process for determining the minimal "claim" of a principal's type on (expected) net surplus, and then allocating the net surplus over all types on the basis of these claims. The claim of a particular type is determined iteratively by deriving the maximum utility that type would have obtained, had it been the highest type, provided that: (i) the upward incentive constraints of all lower types are satisfied; ${ }^{19}$ (ii) output is a non-decreasing function of the type; (iii) all lower types are guaranteed their claims derived in the previous iterations; (iv) buyer breaks even in expectation.

A crucial assumption of our model is that the benefit $S_{i}(q)$ is increasing in the type $i$. This implies (see proof of Lemma 3 in the Appendix) the following important Extension property. Consider any contract $\mu=\left(U_{i}, q_{i}\right)_{1 \leq i \leq N}$ that is feasible for given types $\{1, \ldots, n\}$. Construct the new contract $\nu$ that gives the same transfer-output pairs to types $1, \ldots, n$ and the transfer-output pair of type $n$ to all higher types. This new contract is feasible for any given superset of types that contains types $\{1, \ldots, n\}$. This is a direct consequence of Lemma 1 and the facts that (a) type $n$ and all higher types are bunched, and (b) types $n+1, \ldots, N$ produce higher net surpluses than type $n$ and so they generate profits for the agent. This property will be an important building block for most of our proofs.

Another important property that will be used throughout most of the proofs is the Restriction property. This states that if the assured claim constraint for type $k$ holds with equality in the $n$-assured allocation, with $k<n$, then the restriction of the $n$-assured allocation to the first $k$ types is the $k$-assured allocation. For more details see Lemma 4 in the Appendix.

The above properties, in conjunction with the fact that the objective in each problem

\footnotetext{
${ }^{18}$ The inductive definition presupposes the existence of a solution to the optimization problems $X_{i}$ for $i=1, \cdots, n-1$. Theorem 1 below implies that this is indeed true.

${ }^{19}$ As we will see shortly, satisfying the upward local incentive-compatibility constraints and the monotonicity constraints are enough to ensure feasibility.
} 
$X_{i}$ is to maximize the utility of the highest type, imply also that the downward incentivecompatibility constraints are satisfied by the solution to problem $X_{i}$, for all $i=1, \ldots, N$. The proof of this is more involved than in standard models of adverse selection because at the optimum some types' upward incentive constraints may be satisfied as strict inequalities, while their assured claim constraints be binding. ${ }^{20}$ Thus, the proof that the assured allocation is an incentive compatible mechanism is put in the Appendix (see Lemma 6). Given this and the fact that our main results on the assured allocation are more easily proved by using the above definition, we chose not to include the downward incentive constraints in the definition of the assured allocation.

Focusing on the solution of problem $X_{N}$, first note that the participation constraint is binding, i.e. the corresponding Kuhn-Tucker multiplier is positive and thus the agent makes zero profits in the assured allocation. To understand the intuition behind this, note that if the highest type was not facing the agent's participation constraint, then she could always increase the utility of all types uniformly, and thereby increase her payoff in a an incentive-compatible way. After some straightforward manipulations of the first-order conditions of the problem $X_{N}$, we also have that output $q_{i}^{N}$ is given by

$$
\left(f_{i-1}-g_{i-1}\right) \psi_{i-1}^{\prime}\left(q_{i}^{N}\right)+s_{i} W_{i}^{\prime}\left(q_{i}^{N}\right)+\rho_{i-1}-\rho_{i}=0
$$

where $g_{i} \equiv \sum_{j=1}^{i} \sigma_{j} \leq f_{i}, \rho_{0} \equiv 0$ and $\rho_{N} \equiv 0$, and $\sigma_{j} \geq 0$ and $\rho_{j} \geq 0, j=1, \ldots, N-1$, are the Kuhn-Tucker multipliers of the assured claim constraint $U_{j} \geq V_{j}$ and monotonicity constraint $q_{j+1} \geq q_{j}$, respectively (expressed in terms of the multiplier of the participation constraint). Moreover, the Kuhn-Tucker multiplier of the (local) upward incentive constraint of type $i-1$ (expressed in terms of the multiplier of the participation constraint) is

$$
\mu_{i-1}=f_{i-1}-g_{i-1}
$$

This is intuitive and follows standard arguments. ${ }^{21}$ Due to lower types having an in-

\footnotetext{
${ }^{20}$ If, on the other hand, the upward incentive constraint for some type $i$ holds as equality, then, as in standard adverse selection models, the downward incentive constraint for type $i+1$ follows directly from $\psi_{i}^{\prime} \geq 0$ and the monotonicity constraint $q_{i+1} \geq q_{i}$.

${ }^{21}$ To arrive at (6) simply add up all first-order conditions with respect to $U_{j}, j=1, \ldots, i-1$ and divide across sides by the Kuhn-Tucker multiplier of the participation constraint. (5) is then the first-order condition with respect $q_{i}$ after dividing by the Kuhn-Tucker multiplier of the participation constraint and
} 
centive to mimic higher types, output of each type $j$ maximizes its virtual surplus $\left(f_{j-1}-g_{j-1}\right) \psi_{j-1}(q)+s_{j} W_{j}(q)$ insofar the monotonicity constraints $q_{j+1} \geq q_{j} \geq q_{j-1}$ are slack. If, however, the monotonicity constraints $q_{k^{\prime}} \geq \ldots \geq q_{k}$ are binding, i.e. $\rho_{k}>0, \ldots, \rho_{k^{\prime}-1}>0$, and the monotonicity constraints $q_{k^{\prime}+1} \geq q_{k^{\prime}}$ and $q_{k} \geq q_{k-1}$ are slack, i.e. $\rho_{k-1}=\rho_{k^{\prime}}=0$, then there is bunching between types $k, \ldots, k^{\prime}$ that involves a common output level that maximizes the total virtual surplus of all the types in question $\sum_{j=k}^{k^{\prime}}\left\{\left(f_{j-1}-g_{j-1}\right) \psi_{i-1}(q)+s_{i} W_{i}(q)\right\}$. In any case, the virtual surplus of a type $j$ takes into account that the assured claim constraints of some of the lower types may be binding, relaxing thus the information problem vis-a-vis type $j$.

Note that bunching cannot involve the lowest type, i.e. $\rho_{1}=0$. If it did, then, by definition, $\rho_{1}>0$, and (5) for $i=1$ (and using $f_{0}-g_{0}=\rho_{0}=0$ ) would imply that $q_{1}^{N}<q_{1}^{o}$. In addition, let $j$ be the maximum type involved in the bunching in question (and hence $\rho_{j-1}=\rho_{j}=0$ and $q_{1}^{N}=\ldots=q_{j}^{N}$ ). We have from (5) for $i=j$ that $q_{j}^{N} \geq q_{j}^{o}$ which leads to a contradiction due to $q_{1}^{o}<q_{j}^{o}$. Therefore, $\rho_{1}=0$ and we have from (5) for $i=1$ that $q_{1}^{N}=q_{1}^{o}$. Trivially, then, $V_{1}=W_{1}\left(q_{1}^{o}\right)$. As a corollary we have directly that there is no bunching when $N=2$.

The following Theorem implies that the assured allocation is well-defined.

Theorem 1 The assured allocation exists, is unique and continuous in the prior.

The Extension property discussed above is crucial to obtain admissible mechanisms for the optimization problem defining the assured allocation. Our concavity assumptions together with the Restriction property then guarantee uniqueness. Continuity can be derived using Berge's maximum theorem.

The importance of existence is self-explanatory. Uniqueness is a useful property that will be used in the proof of certain important results (e.g. Theorem 4). The continuity property is particularly desirable because it implies that the proposed allocation is robust to small changes in priors, even if the probability of some types goes to zero.

A natural question is what are the efficiency properties of the assured allocation. We have that:

eliminating $\mu_{i-1}$ by using (6). For more details see Appendix 7 . 
Theorem 2 The assured allocation is undominated within the class of deterministic mechanisms. If there is no bunching in the assured allocation then it is undominated within the class of stochastic mechanisms.

The intuition behind the first part of the above theorem is that if there was a mechanism that dominates the assured allocation, then that mechanism would also be admissible for being the assured allocation, which contradicts its uniqueness. The second part of the theorem, echoing Strauz (2006), relates the optimality of stochastic mechanisms over the assured allocation to the presence of non-monotonic production schedules in the latter allocation.

In Section 5 we will compare in detail the assured allocation with two famous solutions of informed principal problems with common values: the RSW allocation and the neutral optima. The latter are undominated mechanisms and, in our environment, the former allocation has no bunching. One might wonder at this stage whether similar properties hold for the assured allocation. In Lemma 8 in the Appendix we show that under certain conditions the assured allocation also has no bunching. In the second part of the next section we provide an example of an assured allocation with bunching, and show that this allocation is dominated by a stochastic mechanism.

\section{Some Illustrative Examples}

\subsection{Two Types}

Consider the case of two types. Suppose first that

$$
W_{1}\left(q_{1}^{o}\right) \geq W_{2}\left(q_{2}^{o}\right)-\psi_{1}\left(q_{2}^{o}\right)
$$

In this case, the full-information monopoly contract, $m^{M}$, is feasible, ${ }^{22}$ The highest type cannot ensure a higher payoff than $U_{2}^{M}$ subject to feasibility and assured claim constraints. Therefore, the assured allocation coincides with the full-information monopoly contract:

\footnotetext{
${ }^{22}$ The local downward incentive-compatibility constraints are satisfied under the full-information monopoly allocation. To see this, note that for any $i=1, \ldots, N-1, W_{i}\left(q_{i}^{o}\right)+\psi_{i}\left(q_{i}^{o}\right)=S_{i}\left(q_{i}^{o}\right)-C_{i+1}\left(q_{i}^{o}\right)=$ $S_{i+1}\left(q_{i}^{o}\right)-C_{i+1}\left(q_{i}^{o}\right)+S_{i}\left(q_{i}^{o}\right)-S_{i+1}\left(q_{i}^{o}\right) \leq W_{i+1}\left(q_{i+1}^{o}\right)$.
} 
$q_{i}^{2}=q_{i}^{o}, U_{i}^{2}=V_{i}=W_{i}\left(q_{i}^{o}\right), i=1,2$.

Suppose next that

$$
W_{1}\left(q_{1}^{o}\right)<W_{2}\left(q_{2}^{o}\right)-\psi_{1}\left(q_{2}^{o}\right)
$$

In this case, the full-information monopoly mechanism is not incentive compatible: the low productivity type would like to mimic the high productivity type. This implies that the upward incentive constraint is binding in the assured allocation: $U_{1}^{2}=U_{2}^{2}-\psi_{1}\left(q_{2}^{2}\right)$, and by the usual rent-extraction/efficiency trade-off - the output of the high type is distorted upwards, i.e. $q_{2}^{2}>q_{2}^{o}$. From the participation and upward incentive constraints holding as equalities, we can derive that $V_{2}=U_{2}^{2}=\frac{s_{1}}{s_{1}+s_{2}}\left[W_{1}\left(q_{1}^{o}\right)+\psi_{1}\left(q_{2}^{2}\right)\right]+\frac{s_{2}}{s_{1}+s_{2}} W_{2}\left(q_{2}^{2}\right)$ and $U_{1}^{2}=\frac{s_{1}}{s_{1}+s_{2}} W_{1}\left(q_{1}^{o}\right)+\frac{s_{2}}{s_{1}+s_{2}}\left[W_{2}\left(q_{2}^{2}\right)-\psi_{1}\left(q_{2}^{2}\right)\right]$. Moreover, the downward incentive constraint is satisfied, i.e. $U_{1}^{2}+\psi_{1}\left(q_{1}^{o}\right) \leq U_{2}^{2}$, by $\psi_{1}^{\prime}>0$, the upward incentive constraint holding as equality and $q_{2}^{2}>q_{2}^{o}>q_{1}^{o}$. Whether the assured claim constraint is slack depends on the relative likelihood of the two types, $s_{1} / s_{2}$.

To start with, for sufficiently low $s_{1} / s_{2}$, the information problem is not so severe: the highest type is less concerned about leaving information rents than distorting (her) output. This implies that output distortion is not very high, and information rents given to the low type are sufficiently high to ensure that the assured claim constraint is strictly satisfied, i.e. $U_{1}^{2}>V_{1}$ and $\sigma_{1}^{2}=0$. Given that $V_{1}=W_{1}\left(q_{1}^{o}\right)$ and $q_{1}^{2}=q_{1}^{o}$, we thus have that the agent is making losses from the lowest type. As $s_{1} / s_{2}$ increases, the information problem becomes more severe, and output (of the high type) is further distorted and information rents left to the low type are reduced. In this range of priors, we have from (5) for $i=2$ (after using $\rho_{1}=\rho_{2}=0$ ) that $q_{2}^{2}=\arg \max _{q \geq 0}\left\{s_{1} \psi_{1}(q)+s_{2} W_{2}(q)\right\}$. For $s_{1} / s_{2}$ at least equal to a well-defined threshold information rents cannot be reduced further, and the assured claim constraint becomes binding, and so $U_{1}^{2}=V_{1}\left(=W_{1}\left(q_{1}^{o}\right)\right)$. In such a case, we thus have $U_{2}^{2}=W_{2}\left(q_{2}^{2}\right)$, and production of the high type, $q_{2}^{2}$, is given implicitly by $W_{1}\left(q_{1}^{o}\right)=W_{2}\left(q_{2}^{2}\right)-\psi_{1}\left(q_{2}^{2}\right){ }^{23}$ That is, the agent breaks even regardless of the type she faces, and the low type is indifferent between the two offered options. Readers who are familiar with the RSW allocation will immediately recognize that this is the RSW allocation as characterized in Maskin and Tirole (1992).

\footnotetext{
${ }^{23}$ Moreover, $\sigma_{1}^{2}$ is given by solving (5) for $i=2$ with respect to $\sigma_{1}$ (after using $\rho_{1}=\rho_{2}=0$ ), for the given output $q_{2}^{2}$.
} 
It follows that for $s_{1} / s_{2}$ low enough so that the assured claim constraint holds as a strict inequality, the assured allocation dominates the RSW allocation: the highest type selects the assured allocation instead of the (feasible) RSW contract, attaining thus a higher payoff than under the RSW mechanism, while, as we have already noted, the utility of the lowest type is greater than her full-information monopoly payoff, which coincides with her payoff in the RSW allocation. Our Theorem 3 in Section 5.2 is a generalization of this result for any $N$.

As Theorem 2 above shows the assured allocation is an undominated mechanism. The argument here where $N=2$ is very simple: if there was a mechanism that dominates the assured allocation, then that mechanism would also be admissible for being the assured allocation, and the highest type would be able to attain a higher payoff, which is a contradiction. One natural question then is what is the relationship with the Neutral optimum and the core mechanisms, which are also undominated mechanisms. We establish in Theorem 4 in Section 5.3 that for $N=2$ the assured allocation is the only core mechanism. Because neutral optima are always core mechanisms (see Theorem 5 in Myerson (1983)) we therefore have that the assured allocation and the neutral optimum coincide when $N=2$.

\subsection{Three Types: Possibility of Bunching and Assured Alloca- tion Differing from Neutral Optimum}

Moving to the case of three types makes things very complicated quickly. Bunching in the assured allocation becomes now a possibility, and neutral optima, the core and the assured allocations may no longer coincide. The intuition behind this is the following. Consider the case when both upward incentive constraints are violated at the full-information monopoly contract. This implies for the assured allocation that production of the two highest types must be distorted upwards to reduce information rents. Suppose, however, that the upward distortion in the production of the second-highest type is so high that it makes underreporting an attractive option for the highest type. In this case, bunching may emerge in

the assured allocation. Following then similar arguments to Strauz (2006), a stochastic mechanism may dominate the assured allocation, implying that the latter is not a core 
allocation and hence not a neutral optimum. The next example demonstrates.

First, we construct an example where bunching occurs in the assured allocation.

Let $C_{i}(q)=8 q-\theta_{i} \ln (q+1)$ and $S_{i}(q)=\frac{\theta_{i}}{2} q+\theta_{i}\{\ln (q+2)-\ln (2)\}$. Moreover, $\theta_{1}=4, \theta_{2}=7.8, \theta_{3}=8$ with $s_{1}=0.04, s_{2}=0.06$ and $s_{3}=0.9$. We thus have that $\psi_{1}(q)=3.8 \ln (q+1)$ and $\psi_{2}(q)=0.2 \ln (q+1)$. After some simple calculations, one can easily verify that $q_{1}^{o}=0, q_{2}^{o}=2.3695$ and $q_{3}^{o}=2.5616$, whereas $V_{1}=W_{1}\left(q_{1}^{o}\right)=0$ and $W_{2}\left(q_{2}^{o}\right)=5.8559$ and $W_{3}\left(q_{3}^{o}\right)=6.5115$. Note here that $W_{1}\left(q_{1}^{o}\right)<W_{2}\left(q_{2}^{o}\right)-\psi_{1}\left(q_{2}^{o}\right)$ and $W_{2}\left(q_{2}^{o}\right)<W_{3}\left(q_{3}^{o}\right)-\psi_{2}\left(q_{3}^{o}\right)$. That is, the upward incentive constraints are violated by the full-information monopoly contract, and so we should expect both upward incentive constraints to be binding at the assured allocation. We note also that $q_{3}^{o}$ is very close to $q_{2}^{o}$. In addition, $s_{1} / s_{2}$ is relatively large, so that the upward distortion of production of the second highest type is relatively large. Moreover, $\left(s_{1}+s_{2}\right) / s_{3}$ is close to zero, so that the upward distortion of production of the highest type is very small. These last three facts provide conditions for bunching, as we will see next. This example will also be used in our discussion in Section 6 of a particular extensive form game for which the assured allocation is an equilibrium, while the RSW allocation is not.

For these parameters, we have that the assured allocation when only the two lowest types are present, with priors $s_{1} /\left(1-s_{3}\right)$ and $s_{2} /\left(1-s_{3}\right)$, is given by $q_{1}^{2}=q_{1}^{o}, q_{2}^{2}=3.0457$, $U_{1}^{2}=0.0108>V_{1}$ and $U_{2}^{2}=V_{2}=5.5039$. This is different than the RSW allocation where $q_{2}=3.2442$. Note that $q_{2}^{2}$ is given by (5) for $i=2$, after using $\rho_{0}=\rho_{1}=\sigma_{1}=0$ ). To demonstrate the optimality of bunching when $N=3$, consider the solution (denoted by tildas) to problem $\tilde{X}_{3}$, which is as problem $X_{3}$, except that it ignores the monotonicity constraints. This is the solution to problem $X_{3}$ after simply setting in the corresponding Lagrangian $\rho_{1}=\rho_{2}=0$. In this example, we have both upward incentive constraints being binding, and both assured claim constraints being slack. We thus have from (5) for $i=2$ that $\widetilde{q}_{2}^{3}=q_{2}^{2}$. Moreover, we have from (5) for $i=3$ that $\widetilde{q}_{3}^{3}=2.5677$. Note that in this case, using the binding participation and upward incentive constraints, we derive $\widetilde{U}_{1}^{3}=0.8706>V_{1}$ and $\widetilde{U}_{2}^{3}=6.1818>V_{2}$. Therefore, $\sigma_{1}=\sigma_{2}=0$ and $\mu_{i}=f_{i}, i=1,2$, are indeed part of the solution of $\tilde{X}_{3}$. Obviously, this is a solution to problem $\tilde{X}_{3}$, but not to problem $X_{3}$ because it violates the monotonicity constraint $q_{2} \leq q_{3}$. In other words, this mechanism is not feasible because it violates the downward incentive constraint for type 
$i=3$. Consequently, there is bunching in the assured allocation, i.e.

$$
q_{2}^{3}=q_{3}^{3}=\bar{q} \equiv \arg \max _{q \geq 0}\left\{\left(s_{1}+s_{2}\right) \psi_{2}(q)+s_{3} W_{3}(q)+s_{1} \psi_{1}(q)+s_{2} W_{2}(q)\right\}=2.59869220515
$$

Using the binding upward incentive constraints and participation constraint we also have that $U_{1}^{3}=1.3074>V_{1}$ and $U_{2}^{3}=6.1736>V_{2}$; that is, the assured claim constraints are slack under bunching in this example.

Interestingly, the highest type might be able do better than in the above assured allocation with bunching by using a randomized mechanism. The reason echoes the arguments in Strauz (2006). In more detail, by using an appropriate randomized mechanism, the highest type may be able to separate herself from the second highest type by means of relaxing her (downward) incentive constraint. This can be achieved here because, in our example, risk aversion is increasing with productivity, and hence introducing more risk in type $2^{\prime} s$ output makes the latter's output-utility pair less attractive to type $i=3$. Moreover, such a mechanism may also be designed to generate more expected net surplus and hence profits to the agent, which can then be distributed in a lump-sum way to the various types.

Specifically, consider a stochastic mechanism that gives the same expected utility and deterministic output to types $i=3$ and $i=1$, and the same expected utility to type $i=2$, while offering a stochastic output to type $i=2$, such that incentive compatibility is maintained and the expected net surplus produced by type $i=2$ goes up. That is, denoting with $U_{i}$, the expected utility of type $i$, assume that $U_{i}=U_{i}^{3}, i=1,2,3$, $q_{3}=\bar{q}, q_{1}=q_{1}^{o}$. Assume also that the lottery over $q_{2}$ is such that $E W_{2}\left(q_{2}\right)>W_{2}(\bar{q})$ and $E \psi_{2}\left(q_{2}\right) \leq \psi_{2}(\bar{q})$. The former says that the expected net surplus is higher, and the latter implies that the downward incentive constraint of type 3 is (weakly) relaxed, in the randomized mechanism. Finally, if the lottery over $q_{2}$ is such that $E \psi_{1}\left(q_{2}\right) \geq \psi_{1}(\bar{q})$, then the upward incentive constraint of type 1 is still satisfied in the randomized mechanism. ${ }^{24}$

\footnotetext{
${ }^{24}$ We note that the monotonicity constraints under such a stochastic mechanism are $E \psi_{2}\left(q_{2}\right) \leq \psi_{2}(\bar{q})$ and $\psi_{1}\left(q_{1}^{o}\right) \leq E \psi_{1}\left(q_{2}\right)$. The former is the second of the three inequalities in the main text. Note then that we require $E \psi_{1}\left(q_{2}\right) \geq \psi_{1}(\bar{q})$ instead of the remaining monotonicity constraint in the constructed randomized mechanism. The reason is that in the original deterministic mechanism the upward incentive constraint for type 1 is binding, which implies that the corresponding constraint in the stochastic mechanism in question (where expected utilities are kept the same across mechanisms) is satisfied if only if $E \psi_{1}\left(q_{2}\right) \geq \psi_{1}(\bar{q})$. Note that if $E \psi_{1}\left(q_{2}\right) \geq \psi_{1}(\bar{q})$ (which is the last of the three inequalities in question in
} 
If such a mechanism exists, then the highest type can choose an alternative stochastic mechanism that takes some of the excess net surplus (profit of the agent in this case) and distribute it uniformly to all types in the form of utils. This is clearly feasible and makes the highest type strictly better off. In summary, the assured allocation (with bunching) is dominated by a stochastic mechanism if we can find a lottery over $q_{2}$ such that

$$
\begin{aligned}
& E \psi_{1}\left(q_{2}\right) \geq \psi_{1}(\bar{q}) \\
& E W_{2}\left(q_{2}\right)>W_{2}(\bar{q}) \\
& E \psi_{2}\left(q_{2}\right) \leq \psi_{2}(\bar{q})
\end{aligned}
$$

To demonstrate the existence of such a lottery, consider the binary lottery that gives $q_{2}=q_{L}$ with probability $\rho$ and $q_{2}=q_{M}$ with probability $1-\rho$, with $q_{L}<\bar{q}<q_{M}$. In terms of our example, it turns out that the first and third inequalities are satisfied if and only if $\rho=\frac{\ln \left(q_{M}+1\right)-\ln (\bar{q}+1)}{\ln \left(q_{M}+1\right)-\ln \left(q_{L}+1\right)}$, for any given $q_{M}, q_{L}$ and the given $\bar{q}$. Therefore, the problem in hand boils down to finding values for $q_{M}$ and $q_{L}$ such that $E W_{2}\left(q_{2}\right)>W_{2}(\bar{q})$ holds. This is written (in terms of our example) as

$$
\begin{gathered}
\frac{\ln \left(q_{M}+1\right)-\ln (\bar{q}+1)}{\ln \left(q_{M}+1\right)-\ln \left(q_{L}+1\right)}> \\
\frac{\left(q_{M}-\bar{q}\right)(4.1)+(7.8)\left\{\ln (\bar{q}+1)-\ln \left(q_{M}+1\right)+\ln (\bar{q}+2)-\ln \left(q_{M}+2\right)\right\}}{\left(q_{M}-q_{L}\right)(4.1)+(7.8)\left\{\ln \left(q_{L}+1\right)-\ln \left(q_{M}+1\right)+\ln \left(q_{L}+2\right)-\ln \left(q_{M}+2\right)\right\}}
\end{gathered}
$$

One can verify that this inequality is satisfied for $q_{L}=2.598692205125$ and $q_{M}=$ $2.5986924650177,{ }^{25}$ which completes our construction.

The assured allocation in this example is not a core mechanism, and thereby is different from neutral optimum (seen Myerson (1983)). One may wonder what is the relationship between the assured allocation and neutral optima when $N>2$ if there is no bunching in the assured allocation. We show in Theorem 5 that in the absence of bunching, the assured allocation is a neutral optimum.

the main text), then $\bar{q}>q_{1}^{o}$ and $\psi_{1}^{\prime}(q)>0$ imply the remaining monotonicity constraint.

${ }^{25}$ The left hand side equals 0.9999118075 and the right hand side equals 0.9999118031 . Note also that $q_{L}<\bar{q}<q_{M}$. 
To facilitate further understanding of the assured allocation we will compare it next with two famous solutions of informed principal problems with common values: the RSW allocation and the notion of neutral optimum, and by doing so we will derive our main results, Theorems $3-6$.

\section{The RSW Allocation, Neutral Optima and the As- sured Allocation}

We first need to introduce some definitions and review the concepts of RSW and the neutral optima.

\subsection{Safe and Strong Mechanisms}

Adjusting the definitions of a safe mechanism and a strong solution in Myerson (1983) to the way we set up the model here, we have: ${ }^{26}$

Definition 4 A mechanism is safe if it is incentive-compatible and ex post individually rational.

Thus, a safe mechanism is a truth-telling mechanism that the agent is willing to accept to play when she/he knows the principal's type. The principal can successfully implement a safe mechanism as it is feasible regardless of what the agent can infer about the principal who offers such a mechanism.

Definition 5 A mechanism is a strong solution if it is safe and undominated.

A strong solution is an important mechanism because, as Theorem 1 of Myerson (1983) emphasizes, if such a mechanism exists then the principal should implement it despite the fact that he may strictly prefer (given his true type) another feasible mechanism. The reason is that if the agent infers that the principal's type belongs to the set of types that strictly prefer this alternative mechanism to the strong solution, then the former

\footnotetext{
${ }^{26}$ See page 1772 of Myerson (1983) for how to interpret our model in his framework.
} 
mechanism violates the agent's individual rationality given these posterior beliefs on the part of the agent.

Though compelling as a solution concept, a strong solution often does not exist. Recalling Theorems 1 and 2, we have that the assured allocation is a mechanism which, like neutral optima, extends the notion of a strong solution in such a way that a solution always exists and is undominated, but not necessarily safe. The RSW allocation, reviewed next, extends, on the other hand, the notion of a strong solution in such a way that a solution always exists and is safe, but not necessarily undominated. ${ }^{27}$ This highlights the fundamental difference in the concepts of RSW and the assured allocations.

\subsection{Perfect Bayesian Equilibria and the RSW and Assured Al- locations}

The RSW allocation has been studied in terms of the three-stage game $\Gamma$ by Maskin and Tirole (1992). Their Proposition 2 and Theorem 1 will be used here to yield Propositions 1 and 2 below. The following two Remarks facilitate the use of their Proposition 2 and Theorem 1 in our set up.

Remark 2 An undominated mechanism is called an interim efficient allocation in Maskin and Tirole (1992).

Remark 3 Our model satisfies the Sorting Assumption on page 5 of Maskin and Tirole (1992) and the assumptions in their footnote 19, except that we restrict $q$ to be nonnegative. However, even with this restriction, Propositions 2, 4(a) and 5 of Maskin and Tirole (1992) still hold. Thus, the RSW allocation is deterministic and the hypothesis of their Theorem 1 is satisfied as long as RSW exists (see their Remark 3 after their Theorem 1).

In terms of our model, Proposition 2 in Maskin and Tirole (1992) can then be stated as:

\footnotetext{
${ }^{27}$ An extreme example occurs in the model of lender liability studied in Balkenborg (2006) when the informed borrower has all the bargaining power. In the trivial cases where the liability is so low that the borrower can pay it herself, the RSW allocation is a strong solution. In the economically interesting case where the lender has to pay some of the liability, the RSW typically gives all the surplus to the lender, regardless of the type of the borrower. The RSW is dominated by any feasible allocation in that model.
} 
Proposition 1 The $R S W$ allocation $\left(\left(U_{i}^{R S W}, q_{i}^{R S W}\right)\right)_{1 \leq i \leq N}$ is the solution to Programs $\left(R S W_{n}\right)$ defined inductively for $n=1, \cdots, N$ as follows:

$$
\max _{\left(U_{n}, q_{n}\right)} U_{n}
$$

subject to

$$
\begin{aligned}
U_{n-1}^{R S W} & \geq U_{n}-\psi_{n-1}\left(q_{n}\right) \text { provided } n>1 \\
W_{n}\left(q_{n}\right) & \geq U_{n}
\end{aligned}
$$

In particular, the allocation satisfies $W_{n}\left(q_{n}\right)=U_{n}$ and $q_{n}<q_{n+1}$.

Remark 4 Given our assumption on $W_{i}$ and $\psi_{i}$ the solution to this problem exists and is unique. Recalling the above Remark, we thus have that the hypothesis of Maskin and Tirole (1992) Theorem 1 is automatically satisfied.

In contrast to the assured allocation, the RSW allocation is not continuous in priors. To see this, consider the case of $N=2$. In our set-up here we have directly that if the fullinformation monopoly contract $m^{M}$ is not incentive compatible, then the RSW allocation, denoted by $m^{R S W}$, is given by

$$
m_{1}^{R S W}=\left(U_{1}^{R S W}, q_{1}^{R S W}\right)=\left(W_{1}\left(q_{1}^{o}\right), q_{1}^{o}\right)
$$

and

$$
m_{2}^{R S W}=\left(U_{2}^{R S W}, q_{2}^{R S W}\right)=\left(U_{1}^{R S W}+\psi_{1}\left(q_{2}^{R S W}\right), W_{2}^{-1}\left(U_{2}^{R S W}\right)\right)
$$

That is, the low-type seller attains her full-information monopoly option, while the hightype seller's option is such that the low type is indifferent between the two options and the buyer makes zero profits from each and every type. Note that $q_{2}^{R S W}>q_{2}^{o}$. Consequently, there is a discontinuity in the allocation when the prior probability on the low type goes to zero. Namely, the allocation for the high type jumps from $m_{2}^{R S W}$ to the full-information monopoly allocation $m_{2}^{M}$. 
The RSW allocation is the "least-cost-separating allocation" which has received much attention in the signaling and screening literature. Its importance in our set up can be understood by noticing that it is a safe mechanism. Therefore, any type of the principal can guarantee her RSW payoff. Consequently, the principal's utility profile that corresponds to the RSW allocation is a lower bound for the equilibrium utility profile of the principal. Moreover, if there exist beliefs on the part of the agent after observing a deviation from the RSW allocation that ensure the absence of a profitable deviation, then the RSW allocation is an equilibrium of the three-stage game $\Gamma$. This is dealt with in Theorem 1 of Maskin and Tirole (1992). The following proposition is an application of the latter Theorem to our setting (recall our Remark above).

Proposition 2 (Maskin and Tirole) A feasible mechanism $\left(\left(U_{i}, q_{i}\right)\right)_{1 \leq i \leq N}$ is a perfect Bayesian equilibrium allocation of the three-stage game $\Gamma$ if and only if it gives each type of the principal at least his utility in the $R S W$ allocation. In particular, if the $R S W$ allocation is undominated, then it is the only perfect Bayesian equilibrium allocation.

Recalling the definition of a strong solution, we clearly have that if the RSW allocation is undominated then it is a strong solution. In fact, combining the above two propositions with Theorem 1 in Myerson (1983) we show in the Appendix that:

Proposition 3 A strong solution exists if and only if the $R S W$ allocation is undominated.

Therefore, if a strong solution does not exist, then the RSW allocation is dominated and an equilibrium selection problem arises for the mechanism selection game $\Gamma$. In our first example in Section 4 the RSW allocation is indeed dominated by the assured allocation, and hence the latter is an equilibrium of game $\Gamma$. We generalize this finding in our second main result:

Theorem 3 The assured allocation weakly dominates the $R S W$ allocation.

The intuition is as follows. As it is shown in the proof of Theorem 3 in the Appendix, the RSW allocation for every group of first $k$ types is admissible for the $k$-assured allocation. Therefore, the utility of every type under the RSW allocations is at most as high as 
the type's assured claim, with the latter being by definition a lower utility bound on what types can obtain in the assured allocation.

Theorems 1 and 3 and Propositions 2 and 3 imply that the unique assured allocation tracks in a continuous (and computable) way an equilibrium outcome of the game $\Gamma$ which is undominated amongst deterministic mechanisms and coincides with the strong solution whenever the latter exists (and hence with the RSW allocation).

Comparing the RSW and the assured allocations as equilibria of the game $\Gamma$, whenever a strong solution does not exist and hence equilibrium selection is an issue in game $\Gamma$, note first that the principal can successfully implement the RSW allocation because it is a safe mechanism, and so it is feasible regardless of what the agent can infer about the principal who offers such a mechanism. Importantly, however, the assured allocation strictly dominates the RSW allocation. Therefore, following the discussion in Myerson (1983), if the principal could communicate effectively with the agent, then the assured allocation, and not the RSW allocation, could be one mechanism offered by the principal.

Second, note that Theorem 1 implies that if a sequence of priors converges to the distribution which puts all mass on one type, then the assured allocation converges to the full-information monopoly optimum for this type. This is in sharp contrast to the RSW allocation which is prior-independent for a given set of types, but can jump discontinuously if the prior probability on a type goes to zero, as we have discussed earlier. Accordingly, the assured allocation is a more robust equilibrium in such changes of the prior beliefs. However, because the RSW is prior independent for a given set of a priori possible types it could be robust implementable in the sense of Bergemann and Morris (2005). ${ }^{28}$ The RSW yields in our set up the only perfect Bayesian equilibrium satisfying the intuitive criterion of Cho and Kreps (1987) (see Proposition 7 and, for the intuition, Figure 4 and the discussion surrounding it, in Maskin and Tirole (1992)). Yet, as already noted, for instance, by Cho and Sobel (1990) in the conclusions in their paper, the intuitive criterion and related refinement concepts are not continuous and have the drawback of

\footnotetext{
${ }^{28}$ Proving that it is may not merely be a direct application of the results recently derived in Bergemann and Morris (2005), Bergemann and Morris (2008) and the literature they cite. The reason is that in our framework the designer herself has private information. Therefore, firstly, an offered mechanism may have some informational content and, secondly, the type space of the agent will include her prior beliefs about the principal's payoff type, and the type space of the principal will include the prior beliefs of the principal about the agent's prior beliefs.
} 
being sensitive to small changes in the extensive form. A related issue is discussed shortly in Section 6.

Our discussion highlights that an understanding of the robustness properties of mechanisms and equilibrium refinement, on one hand, and how these interact with efficiency and continuity, on the other, is very important. However, it is out of the scope of the current work and must be left for future research. In this paper we will not take a stance on the relative importance of these properties. We do not claim that the assured allocation must be preferred against all other allocations when the RSW allocation is dominated (by the assured allocation). Our contribution in this paper is instead to provide an easily interpreted algorithm for an undominated allocation which is an equilibrium for the 3-stage game in Maskin and Tirole (1992) and, under some conditions discussed next, a neutral optimum. We leave it to the researchers and practitioners to choose which of these two or other allocations to focus on.

\subsection{The Neutral Optimum and the Assured Allocation}

Neutral optimum is an axiomatically founded solution concept (Myerson (1983)) that always exists in environments with finite outcome and type spaces. It cannot be eliminated by any reasonable concept of blocking. ${ }^{29}$ The neutral optimum is not necessarily a strong solution itself. To simplify exposition, we do not state here Theorem 7 in Myerson (1983). However, we will need it in the proof of Theorem 5, in the Appendix, and therefore we will state it there for completeness.

We turn to the comparison of the assured allocation with the neural optimum.

Theorem 4 If $N=2$, then the neutral optimum coincides with the assured allocation and is the unique core mechanism.

Proof. Note that in any core allocation, the lowest type should be getting at least his assured claim. If not, compare with the pooling mechanism which requires both types to produce $q_{1}^{o}$ and yields utility $W_{1}\left(q_{1}^{o}\right)$ to type 1 . This mechanism makes zero profits from the lowest type and positive profits from type 2 (as this type is more productive than the lowest type). It is also incentive compatible for all types due to pooling, and

\footnotetext{
${ }^{29}$ For the original, axiomatic definition see Myerson (1983) or the brief review in Severinov (2009).
} 
hence it is feasible for any superset of whatever the set of winners is in this alternative mechanism (which includes $i=1$ by construction). Therefore, the alternative mechanism is a "blocking" mechanism, which contradicts the assumption that the original mechanism is a core allocation.

We next show that in any core allocation, the highest type should be getting at least his assured claim. If not, compare with the assured allocation for the two types. As we have seen in the discussion of the assured allocation for two types in Section 4.1, (see also Lemma 3 in the Appendix) the agent makes non-negative profits from type $i=2$ under the assured allocation and zero expected profits overall. So this alternative mechanism satisfies the participation constraint regardless of the superset of winners (which includes $i=2$ by construction). Thus it blocks the given mechanism.

Recall now that the assured claim of type 2 is by defiition the maximum utility type 2 can get conditional on offering a feasible mechanism that gives type 1 at least her assured claim. Therefore, the assured allocation coincides with the core allocation when $N=2$. The remaining result follows directly from the result in Myerson (1983) that neutral optima are core allocations, and by the uniqueness of the assured allocation (Theorem 1).

The above result is interesting because, in general, it is very hard to determine the set of all neutral optima, and often even to find a single one. However, in the Theorem above we prove, in effect, uniqueness of the neutral optimum and provide, a simple, computable characterization of it, when $N=2$, via the assured allocation.

The fact that the assured allocation coincides with the core mechanism as defined in Myerson (1985) provides a very strong equilibrium selection argument in favor of the assured allocation, when $N=2$, and emphasizes the importance of the assured allocation for the case of two types.

To proceed to our next result, we need to introduce one more assumption, which makes use of the following definition.

Definition 6 Denote by $q_{i}(\delta)$ for $\delta \in\left[0, f_{i-1}\right]$ the maximizer of $\frac{f_{i-1}-\delta}{s_{i}} \psi_{i-1}(q)+W_{i}(q)$.

Notice that by our previous assumptions the latter function is strictly concave and hence has a unique maximizer $q_{i}(\delta) \geq 0$ or it is never decreasing. Notice also that $q_{i}\left(f_{i-1}\right)=$ $q_{i}^{o}$ and that $q_{i}(\delta)$ is decreasing in $\delta$ whenever $q_{i}(\delta)>0$. 
Assumption B : For all $1 \leq i<j \leq N$ and $\delta \in\left[0, f_{i-1}\right]$, it holds that $q_{i}(\delta) \leq q_{j}(\delta)$, if $q_{i}(\delta)>0$.

Assumption B ensures that there is no bunching in the assured allocation when there are three types or more. It is used in Theorem 5, but not elsewhere. Assumption B is often deployed to ensure no bunching in optimal screening problems with type-dependent outside options. For instance, Assumption B is the counterpart of the Potential Separation assumption in Jullien (2000). Assumption B is needed here because the assured allocation is defined by means of an optimal screening problem with type-dependent reservation utilities. As in Jullien (2000) we can give separate conditions on the priors and the technology which jointly imply Assumption B. These are provided for completeness in Lemma 7 in the Appendix.

Theorem 4 provides also the basic motivation behind Theorem 5. Start from the case with two types. Suppose now that a third, even more productive type of the principal is added. Then, recalling problem $X_{n}$ in Section 3 for $n=N$, we define the assured allocation for the three types as a feasible mechanism that achieves for the third type the maximal payoff conditional on the lower types getting at least their assured claims, which in turn are the payoffs of the lower types if they were the highest types. This construction can be iterated when more and more types of higher efficiency are added to the model. Recall now that there is no bunching in the unique assured allocation when $N=2$. In Lemma 8 in the Appendix we show that Assumption B implies that there is no bunching in the assured allocation for any $N$. Motivated by these findings, and our discussion in Section 4.2 - in particular, that if there is bunching in the assured allocation then it may not be a a neutral optimum - we obtain the final main result of this paper, which is technically the hardest to prove.

Theorem 5 Under Assumption B the assured allocation is a neutral optimum.

The result implies in turn that the assured allocation (without bunching) belongs to the minimal set of "unblocked mechanisms" as defined in Myerson (1983)). This, alongside that the assured allocation dominates the RSW allocation when equilibrium selection arises in game $\Gamma$, provides an equilibrium selection argument in favor of the assured vis-a-vis the RSW allocation when there are more than two types and no bunching in the assured 
allocation. We do not know whether there exists another neutral optimum which is not the assured allocation when in the latter there is no bunching. We strongly conjecture that there are. On the other hand, recalling the second example in Section 4, we know that when there is bunching in the assured allocation, then the assured allocation may not be a neutral optimum. This emphasizes that the assured allocation does not in general coincide with neutral optima.

\section{Market Implementation of the Assured Allocation}

As Proposition 2 and Theorem 3 imply that the assured allocation is a perfect Bayesian equilibrium of the three-stage game $\Gamma$, one may wonder whether there is a dynamic process or game for which the RSW allocation may not be an equilibrium outcome, while the assured allocation is. If such a game exists, this could provide an additional reason for selecting the assured, and not the RSW, allocation as a solution to the informed-principal problem. In this section we provide such a game. In doing so, we also provide an additional link of the assured allocation with the received literature - in particular, the literature on markets with adverse selection.

Consider the following game. There is a unit mass of sellers and $N_{B} \geq 2$ buyers, with each seller and each buyer conforming to the description of "the seller" and "the buyer" in Section 2, respectively. Let $s_{i}$ represent the proportion of sellers of type $i$. Types are the sellers' private information. The game has the following stages:

- At the first stage, the uninformed parties (buyers) post simultaneously and independently incentive-compatible and individually-rational ${ }^{30}$ deterministic direct mechanisms.

- At the second stage, having observed the competitors' postings, each buyer can "withdraw" its contract, i.e. "exit the market", at no cost. Withdrawing a contract means that the buyer decides not to trade with any seller, and thereby makes zero profits.

\footnotetext{
${ }^{30}$ This is without loss of generality as (a) any contract which is not incentive-compatible for some types can be replaced by another mechanism where each type is offered instead its preferred option from the original menu, and (b) any incentive-compatible contract which is not individually-rational for some types can be replaced by another menu of options where each such type is offered its reservation payoff.
} 
- At the last stage, sellers choose an option from the available contracts and trade does take place (i.e. no further market-exits are allowed at this stage). If no contract is available, i.e. if all buyers have exited the market, then sellers receive their reservation utilities.

We call this the "market game". This game is a modification of the model of an insurance market analyzed in Rothschild and Stiglitz (1976) where an exit stage is explicitly added. The idea behind this extension is to allow buyers to react to possible deviations by their competitors, introducing thus forces akin to those behind Wilson's "anticipatory equilibrium" notion.

We restrict attention to priors being such that the assured allocation does not coincide with the RSW allocation. If it did, then it would be a symmetric pure strategy Perfect Bayesian Nash equilibrium outcome of the market game; this is a consequence of being, in this case, a strong solution solution.

We will be referring to type $i$ as "loss-making", if it creates losses for the buyer under the assured allocation, i.e. if $W_{i}\left(q_{i}^{N}\right)<U_{i}^{N}$. Conversely, we will be referring to type $i$ as "profit-making", if it creates positive profits for the buyer under the assured allocation, i.e. if $W_{i}\left(q_{i}^{N}\right)>U_{i}^{N}$.

We then have the following important result:

Theorem 6 Assume that priors are such that all types $i=1, \ldots, N-1$ are loss-making. All buyers posting the assured allocation at the first stage, with buyers sharing the market (i.e. each buyer trading with $s_{i} / N_{B}$ sellers of type $i$, for all $i$ ) is a symmetric pure strategy Perfect Bayesian Nash equilibrium outcome of the market game.

Proof. Let us denote the contract that offers the assured allocation with $\kappa^{N}=$ $\left(U_{i}^{N}, q_{i}^{N}\right)_{i=1}^{N}$. To prove the existence of an equilibrium where this contract is offered by all buyers and no buyer exits the market, we assume that in equilibrium sellers who are indifferent between an option from the assured allocation contract and an option from an alternative contract select the former option. Furthermore, sellers always choose the option which is designed for them. Moreover, we postulate that after a deviating offer by another buyer, the only buyers who exit are those who would have otherwise made losses. 
We prove the theorem by contradiction, and so suppose that there exists a feasible deterministic deviation that makes positive profits. Refer to this deviation as contract $\kappa^{d}=\left(U_{i}^{d}, q_{i}^{d}\right)_{i=1}^{N}$. We will be referring to it simply as the deviation. We will also be saying that a type is "attracted" by the deviating buyer, if the type (weakly) prefers the option offered to it in the first stage by the deviating buyer. Notice that the deviation, being profitable, must have the deviating buyer not exiting the market at the second stage.

Suppose first that the deviation is such that some non-deviating buyers do not exit the market. These buyers are not making losses; otherwise they would have also exited the market. Consider then the mechanism $\kappa^{\prime}=\left(U_{i}^{\prime}, q_{i}^{\prime}\right)_{i=1}^{N}$ that consists of the options that are chosen at the last stage by the sellers from all available contracts. That is,

$$
\left(U_{i}^{\prime}, q_{i}^{\prime}\right)=\left\{\begin{array}{lll}
\left(U_{i}^{N}, q_{i}^{N}\right) & \text { if } & U_{i}^{\prime} \geq U_{i}^{N} \\
\left(U_{i}^{d}, q_{i}^{d}\right) & \text { if } & U_{i}^{\prime}<U_{i}^{N}
\end{array}\right.
$$

This mechanism is feasible and makes strictly positive profits by construction. It also satisfies the assured claim constraints (because no type is worse off following the deviation). This implies that a mechanism $\kappa^{\prime \prime}$ that differs from mechanism $\kappa^{\prime}$ only in that it offers every type a sufficiently small epsilon more utility is also feasible and makes every type strictly better off. This contradicts the fact that the assured allocation is undominated amongst all deterministic mechanisms.

Assume now that the deviation is such that all non-deviating buyers exit the market at the second stage. Since they would have been making losses if they had not exited, it must be that the deviating buyer attracts some profit-making types. By the description of the candidate equilibrium, these types are strictly better off with the option offered to them by the deviating buyer. By assumption, all types $i=1, \ldots, N-1$ are loss-making, which implies by the properties of the assured allocation that the highest type is the only profit-making type. Therefore, this type chooses the option $\left(U_{N}^{d}, q_{N}^{d}\right)$ and $U_{N}^{d}>U_{N}^{N}$. However, the maximum profits that can arise from any incentive-compatible mechanism subject to the condition that the utility of the highest type is at least as high as its utility in the assured allocation is zero. ${ }^{31}$ Thus, contract $\kappa^{d}$ is not a profitable deviation, and the

\footnotetext{
${ }^{31}$ The proof of this is as follows: recalling Lemma 1 we have that all incentive-compatibility constraints in the problem in the main text above can equivalently be replaced by the local incentive-compatibility
} 
proof is comlpete.

A corollary of this theorem is that the assured allocation is an equilibrium of the market game for all priors, conditional on there being only two types. ${ }^{32}$ A natural question is whether there are priors such that all types $i=1, \ldots, N-1$ with $N>2$ are loss-making. The answer is affirmative. In fact, our example in Section 4.2 (of the assured allocation involving bunching) is such an example. Loosely speaking, the highest type will be the only type who is not loss-making, if it is very productive (so that all upward incentive constraints are binding) and is sufficiently more likely than all other types (so that the upward distortion of its output is relatively small). The reason is that in this case, the information problem will not be very severe and the highest type will be willing to leave sufficiently high information rents to (i.e. cross-subsidize) all types below.

Echoing results in the literature on insurance, we can easily show that the RSW will not be an equilibrium outcome of the market game for some priors. To see this, consider momentarily the market game after dropping the second stage. In this game, the RSW is not an equilibrium outcome when higher types are sufficiently more likely than lower types so that there is a profitable pooling contract that attracts all types. This follows directly from single-crossing and the concavity assumptions about the cost and benefit functions. Figure 1 demonstrates this situation for the case of two types (and linear buyers' benefit functions). This figure is the analogue of the corresponding figure that shows the well known case when the RSW is not a Nash equilibrium in the insurance market game (without the second withdrawal stage); see, for instance, Figure III in Rothschild and Stiglitz (1976). The same is clearly also true for the market game as defined earlier (i.e. with the second stage), because such a profitable pooling contract will attract all types

constraints and the monotonicity constraints. Consider the relaxed problem $P$ that results from the latter problem after dropping the local downward incentive constraints. Problem $P$ is a well-defined problem. Note then that the assured allocation and the associated Kuhn-Tucker multipliers (expressed in terms of the multiplier of the participation constraint) from problem $X_{N}$ is a solution to problem $P$ after using the inverse of the multiplier of the participation constraint for the Kuhn-Tucker multiplier of the constraint $U_{N} \geq U_{N}^{N}$ in problem $P$. This follows directly from the fact that for the highest type the assured claim constraint holds by construction as equality, while for all other types the assured claim constraints hold as strict inequalities (and hence the corresponding assured allocation Kuhn-Tucker multipliers are zero). Therefore, maximized profits in problem $P$ are zero. But we know that the assured allocation satisfies also the downward incentive constraints. Thus, the solution in problem $P$ is also a solution to the problem stated in the main text above.

${ }^{32}$ With two types and the assured allocation being different than the RSW allocation, the lowest type is loss-making (recall our discussion in Section 4.1). 
and hence the non-deviating buyers will end up with zero profits and not exit the market. The same construction can easily be extended to the case of more than one types as long as the highest type is sufficiently more likely than lower types.

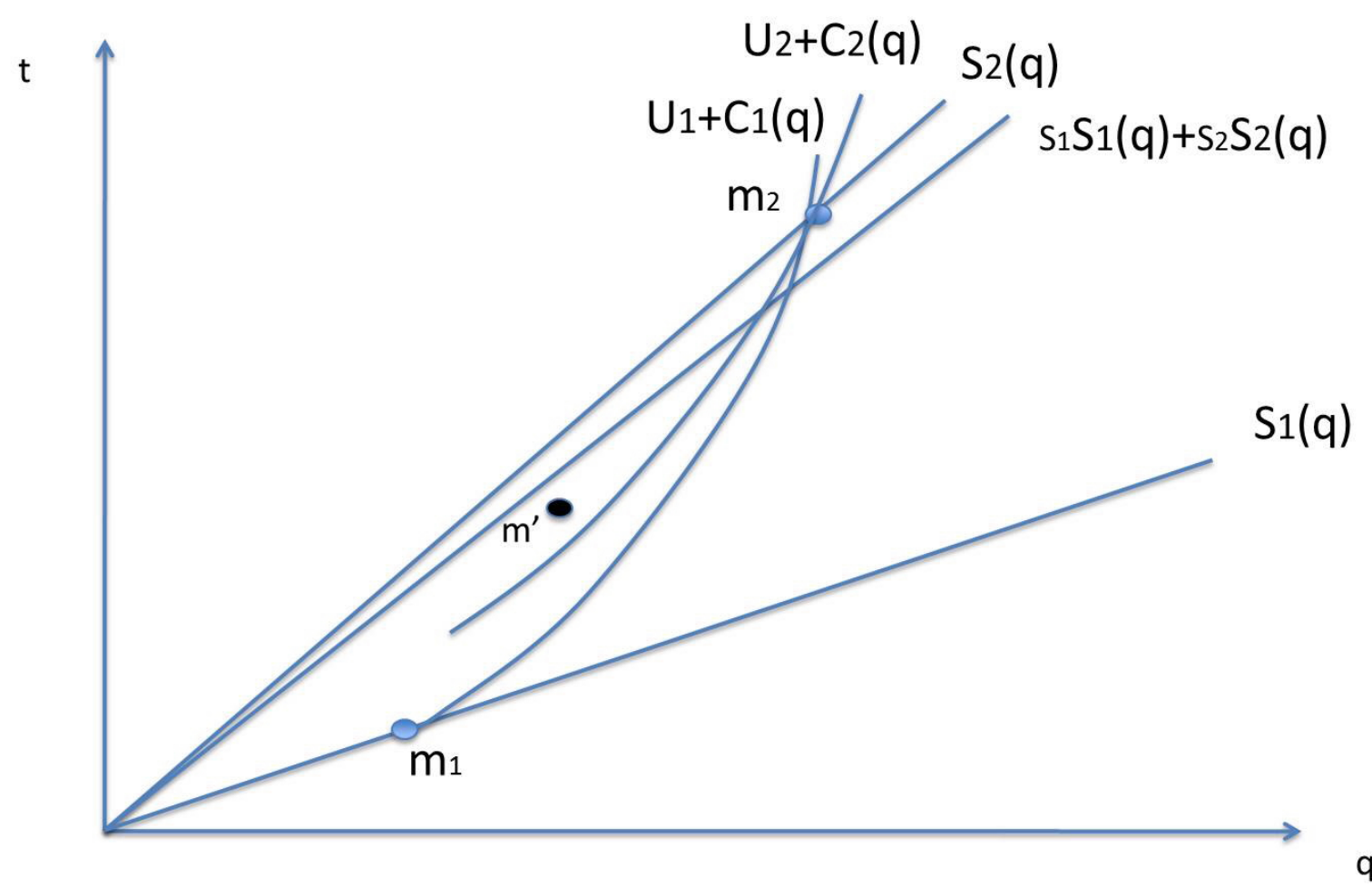

Figure 1: Contract $\mathrm{m}^{\prime}$ is a profitable pooling deviation to RSW allocation $\mathrm{m}$

Given the above discussion, a very important question, for our purposes, is whether there are priors such that the above theorem holds and the RSW allocation is not an equilibrium outcome of the market game. Again, our example in Section 4.2 provides us with such a case. In that example, one can easily see that there is pooling contract that strictly dominates the RSW allocation and makes strictly positive profits. ${ }^{33}$ Therefore, we can have situations where priors are such that an equilibrium outcome of the market game is the assured, but not the RSW contract. The existence of a game for which the

\footnotetext{
${ }^{33}$ To be precise, such mechanism is the pooling contract $\left\{t^{\prime}+0.01, q^{\prime}\right\}$ where $\left\{t^{\prime}, q^{\prime}\right\}=\{20.3848,3.273\}$ is the transfer-output option that would make both types 1 and 3 indifferent between this option and their RSW options. That is, $\left\{t^{\prime}, q^{\prime}\right\}$ is the solution to the system of equations $\left\{t-C_{1}(q)=U_{1}^{R S W}, t-C_{3}(q)=\right.$ $\left.U_{3}^{R S W}\right\}$ where $U_{i}^{R S W}$ denotes the utility of type $i$ under the RSW allocation. For completeness, we note that $U_{i}^{R S W}=W_{i}\left(q_{i}^{R S W}\right)$, with $q_{1}^{R S W}=q_{1}^{o}$, and $q_{2}^{R S W}$ and $q_{3}^{R S W}$ being defined implicitly by $W_{3}\left(q_{3}^{R S W}\right)=W_{2}\left(q_{2}^{R S W}\right)+\psi_{3}\left(q_{3}^{R S W}\right)$ and $W_{2}\left(q_{3}^{R S W}\right)=W_{1}\left(q_{1}^{o}\right)+\psi_{2}\left(q_{2}^{R S W}\right)$.
} 
assured, but not the RSW, allocation is an equilibrium outcome emphasizes the value of the assured allocation, especially when it dominates the RSW allocation.

\section{Conclusions}

In this paper we introduced a specific deterministic mechanism which we called the assured allocation. We showed that it exists, and is unique, undominated (amongst deterministic mechanisms) and robust in small changes in prior beliefs. We also showed that it (weakly) dominates the RSW and coincides with the RSW only when the latter is undominated. If the assured allocation is separating it is a neutral optimum, while we have provided an example where there is bunching in the assured allocation and the latter is not a neutral optimum. We have also shown that when $N=2$ the assured allocation coincides with the neutral optimum and the core mechanism. Therefore, faced with equilibrium selection in the mechanism-selection game in Maskin and Tirole (1992), one could choose the assured allocation as the solution. When $N>2$, the latter argument cannot be made as the assured allocation may not coincide with the core mechanism. We show that when there is no bunching in the assured allocation, then the latter is a neutral optimum and hence a subset of the core. We have also shown that in a particular class of "market games", the assured, but not the RSW, allocation is always an equilibrium. These properties, alongside that the assured allocation dominates the RSW mechanism, could be used to argue in favor of the assured, and not the RSW, allocation as a solution to informed principal problems of the type we study here.

One interesting avenue for future work would be to attempt to generalize the concept of the assured allocation to stochastic mechanisms. This would require a different approach than the one we have employed here. We have shown and extensively used that the surplus attained by each type had it been the highest one, is increasing in the type. However, with stochastic mechanisms this might no longer be always true. The reason is the following. It is true that stochastic mechanisms will in general (weakly) increase the surplus of the highest type, all other things equal. Nevertheless, all other things are not equal. Due to the recursive nature of defining the assured allocation, the constraints that lower types attain at least as much as what they would have attained had they been the highest type, 
may also be more stringent when stochastic mechanisms are allowed.

We expect that our analysis extends to more general environments with multiple agents

and interdependent values provided there remains a clear hierarchy of the types of the principal in terms of attained surplus for contracts which are individually rational and incentive compatible for all types of the principal and all types of the agents. We leave this for future research.

It would be interesting to add ex post participation constraints for the agent (i.e. that he can ex post refuse a contract if the principal decides to take a particular option). Such model modifications, which relax the commitment assumptions used here, could alter the nature of the assured allocation. One could also allow for type-dependent outside options. This would enable the investigation of insurance and franchise contracts. Our analysis does not allow us to handle cases where there is a trade-off between quality and costs. For our analysis, it is important that higher types are unambiguously preferred by the agent. However, interesting applications, such as in the procurement of public services, might require a purchaser who prefers a producer of lower cost-efficiency and higher quality of produced services. All these are very interesting future research projects.

\section{References}

Balkenborg, D. (2006): "Judgment Proofness and Extended Liability in the Presence of Adverse Selection," in Frontiers in the Economics of Environmental Regulation and Liability, ed. by M. Boyer, Y. Hiriart, and D. Martimort, vol. 91, pp. 265 - 290. Ashgate.

Bergemann, D., And S. Morris (2005): "Robust Mechanism Design," Econometrica, $73,1771-1813$.

(2008): "Ex Post Implementation," Games and Economic Behavior, 63(2), 527 $-566$.

Cella, M. (2008): "Informed Principal with Correlation.," Games and Economic Behavior, 64, 433-456. 
Cho, I.-K., And D. Kreps (1987): "Signaling Games and Stable Equilibria," Quarterly Journal of Economics, 102, 179 - 221.

Cho, I.-K., And J. Sobel (1990): "Strategic Stability and Uniqueness in Signaling Games," Journal of Economic Theory, 50, 381 - 413.

Darrough, M., and N. Stoughton (1989): "A Bargaining Approach to Profit Sharing in Joint Ventures," The Journal of Business, 62(2), 237 - 270.

Diasakos, T., and K. Koufopoulos (2009): "Equilibrium and Optimal Mechanisms in Economies with Adverse Selection," unpublished manuscript.

HeLlWig, M. (1987): "Some recent developments in the theory of competition in markets with adverse selection," Eur. Econ. Review, 31, 319 - 325.

Jullien, B. (2000): "Participation Constraints in Adverse Selection Models," Journal of Economic Theory, 93, $1-47$.

Laffont, J.-J., And D. Martimort (2002): The Theory of Incentives - The Principal - Agent Model. Princeton University Press, Princeton and Oxford.

MA, C.-T. A. (1994): "Renegotiation and Optimality in Agency Contracts," The Review of Economic Studies, 61, 109-129.

Maskin, E., and J. Tirole (1990): "The Principal-Agent Relationship With an Informed Principal, I: Private Values," Econometrica, 58, 379 - 409.

(1992): “The Principal-Agent Relationship With an Informed Principal, II: Common Values," Econometrica, 60, 1 - 42.

Myazaki, H. (1977): "The Rat Race and Internal Labor Markets," Bell J. Econ., 8, $394-418$.

Myerson, R. B. (1983): "Mechanism Design by an Informed Principal," Econometrica, 51, $1767-1797$.

(1984): “Two-Person Bargaining Problems with Incomplete Information," Econometrica, 52, $461-487$. 
(1985): "Analysis of Two Bargaining Problems with Incomplete Information," in Game Theoretic Models of Bargaining, ed. by A. Roth, pp. 59 - 69. Cambridge University Press.

Mylovanov, T., and T. TrÖGer (2012): "Informed Principal Problems in Generalized Private Value Environments," Economic Theory, 7, 465-488.

- (2014): "Mechanism design by an informed principal: the quasi-linear privatevalues case," Review of Economic Studies, 81, 1668-1707.

Rothschild, M., And J. Stiglitz (1976): "Equilibrium in Competitive Insurance Markets: An Essay on the Economics of Imperfect Information," The Quarterly Journal of Economics, 90, $629-650$.

Severinov, S. (2009): "An Efficient Solution to the Informed Principal Problem," Journal of Economic Theory, 141, 114 - 133.

Spence, M. (1978): "Product Differentiation and Performance in Insurance Markets," Journal of Public Economics, 10, 427-447.

Stiglitz, J. (1977): "Monopoly, Non-linear Pricing and Imperfect Information: the Insurance Market," The Review of Economic Studies, 44, 407 - 430.

Strauz, R. (2006): "Deterministic Versus Stochastic Mechanisms in Principal-Agent Models," Journal of Economic Theory, 128, 306-314.

von Thadden, E.-L. (1995): "Long-Term Contracts, Short-Term Investment and Monitoring," The Review of Economic Studies, 62, 557-575.

Wilson, C. (1977): "A Model of Insurance Markets with Incomplete Information," Journal of Economic Theory, 16, 167 - 207. 


\section{APPENDIX}

\section{Proof of Lemma 1}

Proof of Lemma 1. First, note, after using the definition of $U_{i}$, that the incentivecompatibility constraints can be re-written, respectively, as

$$
\begin{gathered}
U_{i} \geq U_{j}-\sum_{v=i}^{j-1} \psi_{v}\left(q_{j}\right) \text { for } 1 \leq i<N, i<j \leq N \\
U_{i} \geq U_{j}+\sum_{v=j}^{i-1} \psi_{v}\left(q_{j}\right) \text { for } 1<i \leq N, 1 \leq j<i
\end{gathered}
$$

The proof then follows usual arguments. Specifically, the necessary part follows directly because the local incentive-compatibility constraints for types $i$ and $i+1$ are a subset of all incentive-compatibility constraints. Sufficiency is obtained as follows. (a) The local incentive-compatibility constraints for types $i$ and $i+1$ imply

$$
\psi_{i}\left(q_{i+1}\right) \geq U_{i+1}-U_{i} \geq \psi_{i}\left(q_{i}\right)
$$

and so $q_{i} \leq q_{i+1}$ by the monotonicity of $\psi_{i}$. (b) After forward iteration of $U_{i} \geq U_{i+1}-$ $\psi_{i}\left(q_{i+1}\right)$ we have $U_{i} \geq U_{j}-\sum_{v=i}^{j-1} \psi_{v}\left(q_{v+1}\right)$ for $j>i$. Given $\psi_{v}^{\prime}>0$ and, by monotonicity,

$q_{v+1} \leq q_{j}$ for $i \leq v<j-1$, we get that $U_{i} \geq U_{j}-\sum_{v=i}^{j-1} \psi_{v}\left(q_{j}\right)$. (c) After backward iteration of $U_{i}+\psi_{i}\left(q_{i}\right) \leq U_{i+1}$ we have $U_{j}+\sum_{v=j}^{i} \psi_{v}\left(q_{v}\right) \leq U_{i+1}$ for $j<i$. Given $\psi_{v}^{\prime}>0$ and, by monotonicity, $q_{v} \geq q_{j}$ for $j<v \leq i$, we get that $U_{j}+\sum_{v=j}^{i} \psi_{v}\left(q_{j}\right) \leq U_{i+1}$.

\section{Proofs of Propositions and Theorems 1 - 3}

We first need to prove a number of useful Lemmas. These Lemmas use optimization problems $X_{n}(y), X_{n}^{*}(y)$ and $\widetilde{X}_{n}(y)$.

First, problem $X_{n}(y)$ is derived from problem $X_{n}$ after replacing the participation constraint with $\sum_{i=1}^{n} s_{i}\left(W_{i}\left(q_{i}\right)-U_{i}\right)+s_{n} y \geq 0$ where $y \geq 0$ (that is, $\left.X_{n}=X_{n}(0)\right)$.

Second, let $X_{n}^{*}(y)$ be the more constrained optimization problem which is derived from 
$X_{n}(y)$ by adding the downward incentive constraints

$$
U_{i+1} \geq U_{i}+\psi_{i}\left(q_{i}\right) \quad \text { for } 1 \leq i \leq n-1 . \quad\left(\mathrm{DC}_{i, j}\right)
$$

The significance of this problem comes from Lemma 1 which ensures that a solution to $X_{n}^{*}$ is a feasible mechanism given the set of types $\{1, \ldots, n\}$ for the informed principal problem with $n$ types. We will show in Lemma 6 (stated and proved shortly) that a solution to $X_{n}$ is a solution to $X_{n}^{*}(0)$.

Third, let $\tilde{X}_{n}(y)$ be the less constrained problem which is derived from $X_{n}(y)$ after dropping all the monotonicity constraints $\mathrm{MC}_{i}$. Problem $\tilde{X}_{n}(0)$ characterizes the assured allocation if there is no bunching (see Lemma 8). This problem will be used, for instance, in the proof of Theorem 2.

In both problems $X_{n}^{*}(y)$ and $\tilde{X}_{n}(y)$ the numbers $V_{i}$ used in the assured claim constraints are the maximal values $U_{i}^{i}$ from the problem $X_{i}$.

As we will see, the Lagrangian for the problem $X_{n}(y)$ plays an important role in proving Theorem 5. The Lagrangian for the problem $X_{n}(y)$, with the appropriate multipliers $\sigma_{i}, \mu_{i}, \rho_{i}$ and $\gamma$, is (with $\sigma_{n}+\mu_{n}>0$ )

$$
\begin{aligned}
\mathcal{L} & =\left(\sigma_{n}+\mu_{n}\right) U_{n}+\sum_{i=1}^{n-1} \sigma_{i}\left(U_{i}-V_{i}\right)+\sum_{i=1}^{n-1} \mu_{i}\left(U_{i}-U_{i+1}+\psi_{i}\left(q_{i+1}\right)\right) \\
& +\sum_{i=1}^{n-1} \rho_{i}\left(q_{i+1}-q_{i}\right)+\gamma\left(\sum_{i=1}^{n} s_{i}\left(W_{i}\left(q_{i}\right)-U_{i}\right)+s_{n} y\right) .
\end{aligned}
$$

Notice that $\left(\sigma_{n}+\mu_{n}\right)$ is a weight on the objective function in the Lagrangian and not, strictly speaking, a Lagrange multiplier. Normally one would set $\left(\sigma_{n}+\mu_{n}\right)=1$. However, if one multiplies in a solution to the first-order conditions for the Lagrangian problem all multipliers, including $\left(\sigma_{n}+\mu_{n}\right)$, by the same constant, the optimum is not changed. Rather than fixing $\left(\sigma_{n}+\mu_{n}\right)$ we can hence fix any positive Lagrange multiplier at a suitable value. For us it will be convenient to set $\gamma=1$, once we have shown that $\gamma$ must always be positive. This will ease the comparison between the solutions to the problems $X_{n}(y)$ and $X_{k}(0)$ for $k<n$ below. Note thus that $\sigma_{k}$ and $\mu_{k}$ are determined as Lagrange multipliers for the problem $X_{n}(y)$ while only their sum is determined in the problem $X_{k}(y)$. 
Using $\mu_{0} \equiv \rho_{0} \equiv \rho_{n} \equiv 0$ we can rewrite the Lagrangian as:

$$
\begin{aligned}
\mathcal{L} & =\sum_{i=1}^{n}\left[\sigma_{i}+\left(\mu_{i}-\mu_{i-1}\right)-\gamma s_{i}\right] U_{i}-\sum_{i=1}^{n-1} \sigma_{i} V_{i}+\gamma s_{n} y \\
& +\sum_{i=1}^{n}\left[\mu_{i-1} \psi_{i-1}\left(q_{i}\right)+\gamma s_{i} W_{i}\left(q_{i}\right)+\left(\rho_{i-1}-\rho_{i}\right) q_{i}\right]
\end{aligned}
$$

The first-order conditions are for $i=1, \cdots, n$ :

$$
\begin{gathered}
\sigma_{i}+\left(\mu_{i}-\mu_{i-1}\right)-\gamma s_{i}=0 \\
\mu_{i-1} \psi_{i-1}^{\prime}\left(q_{i}\right)+\gamma s_{i} W_{i}^{\prime}\left(q_{i}\right)+\rho_{i-1}-\rho_{i}=0 .
\end{gathered}
$$

Addition of the former over $i$ yields, since $\sigma_{n}+\mu_{n}>0,0<\sum_{i=1}^{n} \sigma_{i}+\mu_{n}=\gamma \sum_{i=1}^{n} s_{i}$. Hence $\gamma>0$, and so the participation constraint of the agent is binding, and therefore

$$
\sum_{i=1}^{n} s_{i}\left(W_{i}\left(q_{i}\right)-U_{i}\right)+s_{n} y=0
$$

From now on we set $\gamma=1$.

The first-order conditions for $i=1, \cdots, n$ become:

$$
\begin{gathered}
\sigma_{i}+\left(\mu_{i}-\mu_{i-1}\right)-s_{i}=0 \\
\mu_{i-1} \psi_{i-1}^{\prime}\left(q_{i}\right)+s_{i} W_{i}^{\prime}\left(q_{i}\right)+\rho_{i-1}-\rho_{i}=0 .
\end{gathered}
$$

Let $g_{i}=\sum_{j=1}^{i} \sigma_{j}$, with $g_{0} \equiv 0$. The first-order conditions with respect to $U_{i}$ imply, using $f_{i}=\sum_{j=1}^{i} s_{j}$, that

$$
\mu_{i}=f_{i}-g_{i}
$$

Denote a solution to optimization problem $X_{n}(y)$ by $\left(U_{i}^{n}(y), q_{i}^{n}(y)\right)_{1 \leq i \leq n}$. We denote the corresponding multipliers by $\sigma_{i}^{n}(y), \mu_{i}^{n}(y)$, and $\rho_{i}^{n}(y)$. We also write $g_{i}^{n}(y)=$ $\sum_{j=1}^{i} \sigma_{j}^{n}(y)$. Notice that $g_{i}^{n}(y)$ is non-decreasing in $i$. When there is no danger of confusion we will often drop the $(y)$ or even the superscript $n$ in the solution. We can now proceed to the various proofs. 
Lemma 2 The participation constraint of the agent is binding in a solution to the problem $X_{n}(y)$. Moreover, for each type $1 \leq i<n$ either the incentive constraint $I C_{i}$ and/or the assured claim constraint $A C_{i}$ is binding, i.e. $\mu_{i}>0$ and/or $\sigma_{i}>0$.

Proof. The discussion below Formula (8) already showed that the participation constraint must be binding in an optimum; in particular, $\gamma=1$. The second part of the Lemma follows directly from Formula (10) and that $\gamma>0$.

Lemma 3 Suppose type $n$ produces $q_{n}^{n}$ in a solution to $X_{n}$. Then

$$
V_{n+1} \geq V_{n}+\psi_{n}\left(q_{n}^{n}\right) \text { and } W_{n}\left(q_{n}^{n}\right) \geq V_{n}
$$

Proof. Let $\left(\left(U_{i}^{n}, q_{i}^{n}\right)\right)_{1 \leq i \leq n}$ be a solution to the program $X_{n}$. We have thus by definition $U_{n}^{n}=V_{n}$. Consider now the contract $\left(\left(\hat{U}_{i}^{n+1}, \hat{q}_{i}^{n+1}\right)\right)_{1 \leq i \leq n+1}$ for the types $1 \leq i \leq n+1$ defined by $\left(\hat{U}_{i}^{n+1}, \hat{q}_{i}^{n+1}\right)=\left(U_{i}^{n}, q_{i}^{n}\right)$ for $1 \leq i \leq n$ and $\left(\hat{U}_{n+1}^{n+1}, \hat{q}_{n+1}^{n+1}\right)=\left(V_{n}+\psi_{n}\left(q_{n}^{n}\right), q_{n}^{n}\right)$. We show now that this contract is admissible for the problem $X_{n+1}$. Given the definition of $V_{n+1}$, we thus have $V_{n+1} \geq V_{n}+\psi_{n}\left(q_{n}^{n}\right)$. To show admissibility notice first that the contract satisfies by construction all incentive constraints $\mathrm{IC}_{i}$, the monotonicity constraints $\mathrm{MC}_{i}$ and the assured claim constraints $\mathrm{AC}_{i}$.

The participation constraint for the agent is also satisfied. To see the latter, we prove first that $W_{n}\left(q_{n}^{n}\right) \geq V_{n}$ must hold. For $n=1$ this is clear. For $n>1$ we would otherwise obtain from the participation constraint for the problem $X_{n}$ that $\sum_{i=1}^{n-1} s_{i}\left(W_{i}\left(q_{i}^{n}\right)-U_{i}^{n}\right)>$ 0 . So $\left(\left(U_{i}^{n}, q_{i}^{n}\right)\right)_{1 \leq i \leq n-1}$ would be admissible for the problem $X_{n-1}$, give utility at least $V_{n-1}$ to type $n-1$, due to $U_{n-1}^{n} \geq V_{n-1}$ by $\mathrm{AC}_{n-1}$, and have a participation constraint for the agent which is satisfied as a strict inequality. However, this means that we have a solution for problem $X_{n-1}$ in which the participation constraint is slack. This contradicts Lemma 2.

Secondly, we have for any $q$

$$
\begin{aligned}
W_{n+1}(q) & =S_{n+1}(q)-C_{n+1}(q) \geq S_{n}(q)-C_{n+1}(q) \\
& =\left(S_{n}(q)-C_{n}(q)\right)+\left(C_{n}(q)-C_{n+1}(q)\right) \\
& =W_{n}(q)+\psi_{n}(q)
\end{aligned}
$$


and therefore

$$
\begin{aligned}
\sum_{i=1}^{n+1} s_{i}\left(W_{i}\left(\hat{q}_{i}^{n+1}\right)-\hat{U}_{i}^{n+1}\right) & = \\
\sum_{i=1}^{n} s_{i}\left(W_{i}\left(q_{i}^{n}\right)-U_{i}^{n}\right)+s_{n+1}\left(W_{n+1}\left(q_{n}^{n}\right)-\left(V_{n}+\psi_{n}\left(q_{n}^{n}\right)\right)\right) & \geq \\
s_{n+1}\left(W_{n}\left(q_{n}^{n}\right)+\psi_{n}\left(q_{n}^{n}\right)-\left(V_{n}+\psi_{n}\left(q_{n}^{n}\right)\right)\right) & \geq 0
\end{aligned}
$$

The last inequality uses $W_{n}\left(q_{n}^{n}\right) \geq V_{n}$, derived above. Thus, the participation holds.

Lemma 4 Suppose that in a solution $\left(U_{i}^{n}, q_{i}^{n}\right)_{1 \leq i \leq n}$ to $X_{n}(y)$ the $k$-assured claim constraint holds with equality for $1 \leq k<n$. Then

$$
\sum_{i=1}^{k} s_{i}\left(W_{i}\left(q_{i}^{n}\right)-U_{i}^{n}\right)=0
$$

and the restricted solution $\left(U_{i}^{n}, q_{i}^{n}\right)_{1 \leq i \leq k}$ is a solution to $X_{k}(0)$. If, in addition, the assured claim constraint is satisfied as a strict inequality at $k+1$, then we must have $q_{k}^{n}<q_{k+1}^{n}$, i.e. there cannot be bunching between types $k$ and $k+1$.

Proof. Suppose first that $\sum_{i=1}^{k} s_{i}\left(W_{i}\left(q_{i}^{n}\right)-U_{i}^{n}\right)>0$. Then the restriction $\left(\left(U_{i}^{n}, q_{i}^{n}\right)\right)_{1 \leq i \leq k}$ of the solution would satisfy all constraints of the problem $X_{k}(0)$ with the participation constraint being slack. Since type $k$ receives $V_{k}$ in this solution, $\left(U_{i}^{n}, q_{i}^{n}\right)_{1 \leq i \leq k}$ is an optimal solution of $X_{k}(0)$ in which the participation constraint is slack. This contradicts Lemma 2.

Suppose next that

$$
\sum_{i=1}^{k} s_{i}\left(W_{i}\left(q_{i}^{n}\right)-U_{i}^{n}\right)<0
$$

Take a solution $\left(\left(U_{i}^{k}, q_{i}^{k}\right)\right)_{1 \leq i \leq k}$ for the problem $X_{k}(0)$. Consider the new contract defined by

$$
\left(\hat{U}_{i}^{n}, \hat{q}_{i}^{n}\right)= \begin{cases}\left(U_{i}^{k}, q_{i}^{k}\right) & \text { for } \quad i \leq k \\ \left(U_{i}^{n}, q_{i}^{n}\right) & \text { for } \quad i>k\end{cases}
$$

We have

$$
U_{k}^{n}=V_{k}=U_{k}^{k}=\hat{U}_{k}^{n} .
$$


The first equality follows by the assumption of the Lemma, the second by the definition of $V_{k}$ and the third by the above construction. Since the participation constraint for $X_{n}(y)$ is binding in the optimal contract $\left(U_{i}^{n}, q_{i}^{n}\right)_{1 \leq i \leq n}$ and since the participation constraint for $X_{k}(0)$ is binding in the optimal contract $\left(U_{i}^{k}, q_{i}^{k}\right)_{1 \leq i \leq k}$, inequality (14) implies $\sum_{i=1}^{n} s_{i}\left(W_{i}\left(\hat{q}_{i}^{n}\right)-\hat{U}_{i}^{n}\right)+s_{n} y>0$. Therefore, the above contract satisfies the participation constraint of problem $X_{n}(y)$ strictly. By construction all assured claim constraints and all incentive constraints $\mathrm{IC}_{i}$ are satisfied as well as the monotonicity constraints $\mathrm{MC}_{i}$ for $1 \leq i \leq n$ possibly with the exception of $\hat{q}_{k+1}^{n} \geq \hat{q}_{k}^{n}$. However, also the latter inequality holds.

To see this, note

$$
V_{k}+\psi_{k}\left(\hat{q}_{k+1}^{n}\right)=\hat{U}_{k}^{n}+\psi_{k}\left(\hat{q}_{k+1}^{n}\right) \geq \hat{U}_{k+1}^{n} \geq V_{k+1} \geq V_{k}+\psi_{k}\left(q_{k}^{k}\right)
$$

which implies by the monotonicity of $\psi_{k}$ that $\hat{q}_{k+1}^{n} \geq q_{k}^{k}=\hat{q}_{k}^{n}$. The equality follows from (15). The first inequality is $\mathrm{IC}_{k}$ after using the definition of the new contract and, once again, (15). The second inequality combines the definition of the new contract with either $\mathrm{AC}_{k+1}$ if $\kappa+1<n$, or $U_{n}^{n} \geq V_{n}$ if $\kappa+1=n$. The last inequality follows from Lemma 3 .

Therefore, $\left(\left(\hat{U}_{i}, \hat{q}_{i}\right)\right)_{1 \leq i \leq n}$ is admissible for the problem $X_{n}(y)$ with a slack participation constraint. Since $\hat{U}_{n}^{n}=U_{n}^{n}$, it is also an optimal solution for this problem, again in contradiction to Lemma 2.

Thus the equality (13) must hold. In consequence, $\left(U_{i}^{n}, q_{i}^{n}\right)_{1 \leq i \leq k}$ is admissible for the problem $X_{k}(0)$ and, since $U_{k}^{n}=V_{k}$, it is an optimal solution for this problem.

Finally, we prove the second part of the Lemma. Suppose that the assured claim constraint is satisfied as a strict inequality at $k+1$, i.e. $U_{k+1}^{n}>V_{k+1}$, and that $q_{k}^{n}=q_{k+1}^{n}$. Since $q_{k}^{n}$ is part of a solution to $X_{k}(0)$, Lemma 3 implies

$$
U_{k+1}^{n}>V_{k+1} \geq V_{k}+\psi_{k}\left(q_{k}^{n}\right)=U_{k}^{n}+\psi_{k}\left(q_{k+1}^{n}\right)
$$

which contradicts the incentive constraint $\mathrm{IC}_{k}$.

Lemma 5 Assume that the solution $\left(U_{i}^{n}, q_{i}^{n}\right)_{1 \leq i \leq n}$ to the optimization problem $X_{n}(y)$ for any $1 \leq n \leq N$ exists. Then the solution $\left(U_{i}^{n}, q_{i}^{n}\right)_{1 \leq i \leq n}$ to the optimization problem $X_{n}(y)$ 
is unique. Moreover, the corresponding multipliers $\sigma_{i}^{n}, \mu_{i}^{n}$ and $\rho_{i}^{n}$ as defined above (see formula (7)) are unique up to the choice of the terms in $\sigma_{n}^{n}+\mu_{n}^{n}$.

Proof. The proof is by induction. The claim is clearly true for $n=1$. Suppose it holds for $n-1 \geq 1$. In the problem $X_{n}(y)$ let $k<n$ be the largest index for which the assured claim constraint is binding. That is, $\sigma_{k}^{n}>0$ and $\sigma_{i}^{n}=0$ for any $k<i \leq n$. (Set $k=0$ if no assured claim constraint is binding. The remaining statements in this paragraph are then vacuously true.) Let $\left(U_{i}^{k}, q_{i}^{k}\right)_{1 \leq i \leq k}$ be the solution to the problem $X_{k}(0)$, which is by assumption unique. Also the Lagrange multipliers $\sigma_{i}^{k}, \mu_{i}^{k}$, and $\rho_{i}^{k}$ of the latter problem are unique, up to the choice of the terms in $\sigma_{k}^{k}+\mu_{k}^{k}$. Let $\left(U_{i}^{n}, q_{i}^{n}\right)_{1 \leq i \leq n}$ be the solution to the problem $X_{n}(y)$, and let $\sigma_{i}^{n}, \mu_{i}^{n}$, and $\rho_{i}^{n}$ be corresponding Lagrange multipliers. By Lemma $4\left(U_{i}^{n}, q_{i}^{n}\right)_{1 \leq i \leq k}$ is a solution to $X_{k}(0)$ and by the assumed uniqueness we have $U_{i}^{n}=U_{i}^{k}$ and $q_{i}^{n}=q_{i}^{k}$ for $1 \leq i \leq k$. From definition of $k$ and the second part of Lemma 4 , we know that $\mathrm{MC}_{k}$ is slack, i.e. $\rho_{k}^{n}=0\left(=\rho_{k}^{k}\right)$. The first-order conditions for the problem $X_{k}(0)$ are hence a subset of the set of first-order conditions for the problem $X_{n}(y)$. Thus $\sigma_{i}^{k}=\sigma_{i}^{n}$, $\mu_{i}^{k}=\mu_{i}^{n}$ and $\rho_{i}^{k}=\rho_{i}^{n}$ for $i<k$ and $\sigma_{k}^{k}+\mu_{k}^{k}=\sigma_{k}^{n}+\mu_{k}^{n}$ by the induction assumption.

It remains to show the uniqueness of $\mu_{k}^{n}, \sigma_{k}^{n},\left(U_{i}^{n}, q_{i}^{n}, \sigma_{i}^{n}, \mu_{i}^{n}, \rho_{i}^{n}\right)_{k+1 \leq i \leq n-1}, U_{n}^{n}, q_{n}^{n}$ and $\sigma_{n}^{n}+\mu_{n}^{n}$. We do this first for a given 'bunching pattern'. By the latter we mean that the set $B$ of indices $i$ for which $q_{i}^{n}=q_{i+1}^{n}$ holds is fixed. The binding incentive constraints $I C_{i}$ for $k<i<n$ (recall the definition of $k$ and the second part of Lemma 2) give

$$
U_{i}^{n}=U_{n}^{n}-\sum_{j=i}^{n-1} \psi_{j}\left(q_{j+1}^{n}\right)
$$

where $U_{n}^{n}$ is by definition the same in all solutions of $X_{n}(y)$. Since $\sigma_{i}^{n}=0$ for $k<i<n$ by assumption, $\mu_{i}^{n}$ for $k \leq i<n$ and $\sigma_{n}^{n}+\mu_{n}^{n}$ are uniquely determined by formula (12) and depend only on $g_{k}^{n}$ or, equivalently, $\sigma_{k}^{n}$. In particular, $\mu_{i}^{n}=f_{i}-g_{k}^{n}$. For any $i \notin B$ we have that $\mathrm{MC}_{i}$ is slack, i.e. $\rho_{i}^{n}=0$. Partition the set of indices $\{k+1, \cdots, n\}$ into maximally connected sets $J$ such that the monotonicity constraint is binding for any two adjacent indices $i, i+1$ in $J$. Thus a set $J=\left\{i_{1} \leq i \leq i_{2}\right\}$ is in the partition if $i_{1}-1, i_{2} \notin B$ and for all $i \in J$ it holds that $(i \in B \Leftrightarrow i+1 \in J)$. (Notice that $J=\{i\}$ is a set in the partition if neither $q_{i-1}^{n}=q_{i}^{n}$ nor $q_{i}^{n}=q_{i+1}^{n}$.) Summing the first-order conditions (11) over all $i \in J$ 
gives the equation

$$
\left.\sum_{j \in J}\left(\mu_{j-1}^{n} \psi_{j-1}^{\prime}(q)+s_{j} W_{j}^{\prime}(q)\right)\right)=0
$$

in which no non-zero $\rho_{i}^{n}$ occur and from which $q_{i}^{n}$ for any $i \in J$ can be inferred uniquely from $\left\{\mu_{i-1}^{n}\right\}_{i \in J}$ and hence $g_{k}^{n}$ by the implicit function theorem because the derivative of the left hand side of the equation is strictly negative with respect to both $q$ and $g_{k}^{n}$. It follows moreover that $q_{i}^{n}$ is a decreasing function of $g_{k}^{n}$ with strictly negative derivative. Starting with the lowest index in $J=\left\{i_{1} \leq i \leq i_{2}\right\}$ one can then infer the $\rho_{i_{1}}^{n}, \rho_{i_{1}+1}^{n}, \cdots, \rho_{i_{2}-1}^{n}$ inductively from the first-order conditions (11).

We now claim that the left-hand term in the participation constraint (9) is, with the variables determined as just described, strictly increasing in $g_{k}^{n}$. To show this we prove that its derivative with respect to $g_{k}^{n}$ is strictly positive except in a single point. The terms on the left-hand side in (9), which depend on $g_{k}^{n}$, are, using (16) and, once again, the definition of $k$ and Lemma 4 ,

$$
\begin{aligned}
\sum_{i=k+1}^{n} s_{i}\left(W_{i}\left(q_{i}^{n}\right)-U_{i}^{n}\right) & =\sum_{i=k+1}^{n} s_{i}\left(W_{i}\left(q_{i}^{n}\right)-U_{n}^{n}+\sum_{j=i}^{n-1} \psi_{j}\left(q_{j+1}^{n}\right)\right) \\
& =\sum_{i=k+1}^{n}\left(s_{i}\left(W_{i}\left(q_{i}^{n}\right)-U_{n}^{n}\right)+\left(f_{i-1}-f_{k}\right) \psi_{i-1}\left(q_{i}^{n}\right)\right)
\end{aligned}
$$

where the last equality follows by collecting terms and using the definition of the cumulative probability $f_{j}$. Differentiating, and using the equation (11) and that, by $g_{i}^{n}=g_{k}^{n}$ for $k \leq i<n$ and equation (12), $\mu_{i}^{n}=f_{i}-g_{k}^{n}=f_{i}-f_{k}+\mu_{k}^{n}$ for $k<i<n$, we have (recall $\left.\rho_{k}^{n}=0.\right)$

$$
\begin{aligned}
& \sum_{i=k+1}^{n}\left(s_{i} W_{i}^{\prime}\left(q_{i}^{n}\right)+\left(f_{i-1}-f_{k}\right) \psi_{i-1}^{\prime}\left(q_{i}^{n}\right)\right) \frac{d q_{i}^{n}}{d g_{k}^{n}}=\sum_{i=k+1}^{n}\left(-\mu_{k}^{n} \psi_{i-1}^{\prime}\left(q_{i}^{n}\right)+\rho_{i}^{n}-\rho_{i-1}^{n}\right) \frac{d q_{i}^{n}}{d g_{k}^{n}} \\
& =-\mu_{k}^{n} \sum_{i=k+1}^{n} \psi_{i-1}^{\prime}\left(q_{i}^{n}\right) \frac{d q_{i}^{n}}{d g_{k}^{n}}-\sum_{i=k}^{n-1} \rho_{i}^{n}\left(\frac{d q_{i+1}^{n}}{d g_{k}^{n}}-\frac{d q_{i}^{n}}{d g_{k}^{n}}\right)=-\mu_{k}^{n} \sum_{i=k+1}^{n} \psi_{i-1}^{\prime}\left(q_{i}^{n}\right) \frac{d q_{i}^{n}}{d g_{k}^{n}}>0
\end{aligned}
$$

where the last equality follows from $\mu_{k}^{n}>0$ and $\frac{d q_{i}^{n}}{d g_{k}^{n}}<0$ for $k<i<n$. The former is true whenever $g_{k}^{n}<f_{k}$ (recall (12) and that $g_{k}^{n} \in\left[0, f_{k}\right]$ ), while the latter is due to the monotonicity properties of the $\psi_{i}$ and $W_{i}$. Thus the derivative is strictly positive except 
when $g_{k}^{n}=f_{k}$, which proves our claim. Therefore, there can be at most one value of $g_{k}^{n}$ for which the participation constraint is satisfied. It follows that there can only be one solution (including the Lagrange multipliers) for each bunching pattern.

Suppose, finally, that we have two different solutions to the optimization problem $X_{n}(y)$ (excluding the Lagrange multipliers) with possibly different bunching patterns. Any convex combination of the two solutions is also a solution because the optimization problem is convex. Since there are infinitely many convex combinations and only finitely many bunching patterns, we can find two different solutions with the same bunching pattern, which contradicts the above finding. This concludes the proof.

We start with the proof of Theorem 1. In fact, when it comes to the existence proof, we will prove the more general result that a solution to problem $X_{n}(y)$ exists for any $y \geq 0$. Proof of Theorem 1. We first prove existence. Denoting a solution to optimization problem $X_{n}(y)$ by $\left(U_{i}^{n}(y), q_{i}^{n}(y)\right)_{1 \leq i \leq n}$, we have the following:

The proof of existence is by induction on the number of types $n$. Our assumptions imply that the first-best level $q_{1}^{0}$ exists and is finite. Moreover, type 1 has a non-negative utility $V_{1}=W_{1}\left(q_{1}^{0}\right)$ in this mechanism. Therefore a solution exists for $n=1$. Suppose a solution exists for all $i=1, \cdots, n$ and that all $V_{i}$ are non-negative. Then there is a feasible mechanism for the optimization problem where the most productive type $n+1$ gets at least $V_{n} \geq 0$. Namely, take an optimal mechanism for type $n$ and extend it such that type $n+1$ produces $q_{n+1}=q_{n}^{n}(y)$ and receives utility $U_{n+1}$ such that the relevant local incentive-compatibility constraints are satisfied, i.e. $\psi_{n}\left(q_{n}^{n}(y)\right) \leq U_{n+1}-V_{n} \leq \psi_{n}\left(q_{n+1}\right)$. Obviously, this is feasible by the monotonicity property of $\psi_{n}$. Consider next a sequence of feasible mechanisms for which $U_{n+1}$ converges to the (non-negative) supremum of all possible values of $U_{n+1}$ in any feasible mechanism of the problem. Suppose that for some subsequence the value of $q_{i}$ for some $1 \leq i \leq n+1$ goes to infinity. Then the surplus $W_{i}\left(q_{i}\right)$ of type $i$ goes to minus infinity by assumption. Since all types $j=1, \cdots, n$ have utility at least $V_{j} \geq 0$ in each mechanism and since the participation constraint must hold, $U_{n+1}$ must go to $-\infty$, contradicting $U_{n+1} \geq 0$. By selecting a subsequence we can assume that all $q_{i}$ in the sequence converge to a finite value and hence the surplus $\sum_{i=1}^{n+1} s_{i} W_{i}\left(q_{i}\right)$ converges to a finite value. Because each type $j=1, \cdots, n$ has utility at least $V_{j} \geq 0$ in each mechanism in the sequence, $U_{n+1}$ is bounded from above due to the participation 
constraint. Hence a maximizing mechanism exists.

Uniqueness follows from existence and Lemma 5.

We finish with the proof of continuity. Berge's maximum theorem implies by induction that the claims $V_{1}, \cdots, V_{n-1}$ are continuous in the prior. By the same theorem $V_{n}$ is continuous and the $n$-assured allocation upper hemi-continuous. Because the latter is unique, it is therefore continuous.

The proceed with the following important Lemma:

Lemma 6 The solution $\left(\left(U_{i}^{n}, q_{i}^{n}\right)\right)_{1 \leq i \leq n}$ for problem $X_{n}$ is also a solution to the complete problem $X_{n}^{*}$, which therefore exists and is unique.

Proof. To prove this we need to show that the solution $\left(\left(U_{i}^{n}, q_{i}^{n}\right)\right)_{1 \leq i \leq n}$ for problem $X_{n}$ satisfies the downward incentive constraints. For type $1 \leq i<n$ we distinguish two cases: i) The incentive constraint $\mathrm{IC}_{i}$ holds as equality. Then, by using also the monotonicity constraint $\mathrm{MC}_{i}$ we have

$$
U_{i}^{n}=U_{i+1}^{n}-\psi_{i}\left(q_{i+1}^{n}\right) \leq U_{i+1}^{n}-\psi_{i}\left(q_{i}^{n}\right)
$$

and hence $\left(\mathrm{DC}_{i}\right)$ is satisfied.

ii) The $\mathrm{IC}_{i}$ holds as a strict inequality (and hence $\mu_{i}=0$ ). Then, by Lemmas 4 and $2, \mathrm{AC}_{i}$ is binding. Therefore, by Lemma 3, and $\mathrm{AC}_{i+1}$ and the definition of $V_{n}=U_{n}^{n}$ we have

$$
U_{i+1}^{n} \geq V_{i+1} \geq V_{i}+\psi_{i}\left(q_{i}^{i}\right)=U_{i}^{n}+\psi_{i}\left(q_{i}^{i}\right)
$$

Note thus that if $q_{i}^{i} \geq q_{i}^{n}$, then the incentive constraint $\left(\mathrm{DC}_{i}\right)$ holds. In fact, we show next that $q_{i}^{i}=q_{i}^{n}$. First, we have from Theorem 1 the uniqueness of the solution to problems $X_{i}$ and $X_{n}$. Second, we have, from Lemma 4 and that $\mathrm{AC}_{i}$ is binding, that the solution to problem $X_{i}$ is part of the solution to problem $X_{n}$, and hence $q_{i}^{i}=q_{i}^{n}$.

Thus, the solution $\left(\left(U_{i}^{n}, q_{i}^{n}\right)\right)_{1 \leq i \leq n}$ for problem $X_{n}$ is admissible for problem $X_{n}^{*}$. Problem $X_{n}^{*}$ is more restricted than problem $X_{n}$. Hence, existence and uniqueness of the solution to $X_{n}$ (Theorem 1) implies existence and uniqueness of the solution to $X_{n}^{*}$.

We next prove Theorem 2 To do so the following remark is in order. 
Remark 5 In the same fashion, it can be shown that Theorem 1 for problem $X_{n}(y)$ and Lemmas 2-5 (all proved earlier in this Appendix) apply to optimization problem $\tilde{X}_{n}(y)$ as well.

Proof of Theorem 2. First, note from the above Lemma and Lemma 1 that the assured allocation is incentive compatible, and thereby a feasible (deterministic) mechanism. Suppose now the $n$-assured allocation is dominated by another mechanism. Then this alternative allocation is also a solution to $X_{n}(0)$ and hence, by uniqueness (Theorem 1), identical to the $n$-assured allocation. The proof of the first part is complete after setting $n=N$.

We prove next the second part of the theorem. After using (with a slight abuse of notation) $U_{i}=t_{i}-\int C_{i}(q) d \mu_{i}(q)$, the incentive-compatibility and participation constraints can be re-written, respectively, as

$$
\begin{gathered}
U_{i} \geq U_{j}-\sum_{v=i}^{j-1} \int \psi_{v}(q) d \mu_{j}(q) \text { for } 1 \leq i<N, i<j \leq N, \\
U_{i} \geq U_{j}+\sum_{v=j}^{i-1} \int \psi_{v}(q) d \mu_{j}(q) \text { for } 1<i \leq N, 1 \leq j<i, \\
\sum_{i=1}^{N} s_{i}\left(\int W_{i}(q) d \mu_{i}(q)-U_{i}\right) \geq 0 .
\end{gathered}
$$

Suppose now that the assured allocation $\left(\left(U_{i}, q_{i}\right)\right)_{1 \leq i \leq N}$ does not entail bunching, in which case it is admissible for the more restricted problem $\tilde{X}_{N}$. By Remark 5 , the assured allocation is thus the unique solution for problem $\tilde{X}_{N}$. The same argument as in the proof of the first part of this theorem shows that the assured allocation is undominated within the class of all deterministic mechanisms where only the agent's participation constraint $(\mathrm{PC})$, the assured claim constraints $\left(\mathrm{AC}_{i}\right)$ and the upward incentive constraints $\left(\mathrm{IC}_{i}\right)$ are imposed (but not the monotonicity constraints $\mathrm{MC}_{i}$ ).

Consider now a feasible stochastic mechanism $\hat{\kappa}=\left(\hat{U}_{i}, \hat{\mu}_{i}(q)\right)_{1 \leq i \leq N}$ that satisfies the stochastic version of the above constraints, and which dominates the assured allocation. In particular, any stochastic mechanism satisfying all constraints of (17) could be allowed for.

Consider the deterministic outputs $\left(\hat{q}_{i}\right)_{1 \leq i \leq N}$ where $\hat{q}_{i}=\int q d \hat{\mu}_{i}(q)$. We have by Jensen's 
inequality and the strict concavity of $W_{i}(q)$ that $W_{i}\left(\hat{q}_{i}\right)>\int W_{i}(q) d \hat{\mu}_{i}(q)$. Let

$$
S=\sum_{i=1}^{N} s_{i}\left(W_{i}\left(\hat{q}_{i}\right)-\int W_{i}(q) d \hat{\mu}_{i}(q)\right) / N>0
$$

be the associated per-type gain in expected surplus. Consider the deterministic mechanism $\kappa^{\prime}=\left(\hat{U}_{i}+S, \hat{q}_{i}\right)_{1 \leq i \leq N}$, which dominates the stochastic mechanism $\hat{\kappa}$ and thereby the assured allocation. Clearly, the new deterministic mechanism $\kappa^{\prime}$ satisfies by construction the assured claim constraints and the participation constraint of the agent. From Jensen's inequality and the concavity of $\psi_{i}(q)$ we have that $\psi_{i}\left(\hat{q}_{i}\right) \geq \int \psi_{i}(q) d \hat{\mu}_{i}(q)$. Thus, the mechanism $\kappa^{\prime}$ satisfies also the upward incentive constraints $\left(\mathrm{IC}_{i}\right)$. It follows that the unique assured allocation is identical with the mechanism $\left(\hat{U}_{i}+S, \hat{q}_{i}\right)_{1 \leq i \leq N}$ and yields the same payoffs for all types of the principal and for the agent as the stochastic mechanism $\hat{\kappa}$. We conclude that the assured allocation is undominated within the class of stochastic mechanisms.

We now turn to the proofs of Proposition 3.

Proof of Proposition 3. From the definition of a strong solution and Proposition 2 we know that if the RSW allocation is undominated, it is a strong solution and hence a strong solution exists. To prove the converse we notice again that the RSW allocation is safe. It is hence, in the terminology of Myerson (1983), incentive compatible given $S$ for any subset $S$ of types of the principal. Theorem 1 of Myerson (1983) implies hence that a strong solution, if it exists, dominates weakly any safe mechanism, in particular the RSW allocation. Suppose a strong solution exists and strictly dominates the RSW allocation. Then there exists a type of the principal who does not get in the RSW his highest possible payoff amongst all mechanisms which are incentive compatible and yield non-negative payoffs to the agent conditional on every type of the principal. However, this contradicts the definition of the RSW allocation in Maskin and Tirole (1992), p. 11 (which appears here as Proposition 1 for convenience). Thus the RSW yields the same payoffs for each type of the principal as the given strong solution and is hence undominated.

Proof of Theorem 3. For $n=1, \ldots, N$ consider the following allocations. Let $\left(\left(U_{i}^{R S W}, q_{i}^{R S W}\right)\right)_{1 \leq i \leq n}$ be the RSW allocation for the restricted typeset $\{1, \ldots n\}$. Let 
$\left(\left(U_{i}^{n}, q_{i}^{n}\right)\right)_{1 \leq i \leq n}$ be a solution to the problem $X_{n}$. Let $\left(\left(\hat{U}_{i}^{n}, \hat{q}_{i}^{n}\right)\right)_{1 \leq i \leq n}$ be the contract which satisfies $\left(\hat{U}_{i}^{n}, \hat{q}_{i}^{n}\right)=\left(U_{i}^{n-1}, q_{i}^{n-1}\right)$ for any $1 \leq i<n$ and where $\left(\hat{U}_{n}^{n}, \hat{q}_{n}^{n}\right)$ solves the following optimization problem $Z_{n}$ :

$$
\max _{\left(U_{n}, q_{n}\right)} U_{n}
$$

subject to

$$
\begin{aligned}
U_{n-1}^{n-1} & \geq U_{n}-\psi_{n-1}\left(q_{n}\right) \text { for } n>1 \\
W_{n}\left(q_{n}\right) & \geq U_{n}
\end{aligned}
$$

We prove by induction over $n$ that a) $\left(U_{n}^{R S W}, q_{n}^{R S W}\right)$ is admissible for the problem $Z_{n}$ and hence satisfies $U_{n}^{R S W} \leq \hat{U}_{n}^{n}$, and b) $\left(\left(\hat{U}_{i}^{n}, \hat{q}_{i}^{n}\right)\right)_{1 \leq i \leq n}$ is admissible for the problem $X_{n}$ and hence satisfies that $\hat{U}_{n}^{n} \leq U_{n}^{n}=V_{n}$. a) and b) imply directly that $U_{n}^{R S W} \leq V_{n}$, which proves the theorem.

For $n=1$ all three solutions coincide and so our claims hold. Suppose that they hold for $n-1 \geq 1$. To prove claim a) for type $n$, notice that $U_{n-1}^{R S W} \leq \hat{U}_{n-1}^{n-1} \leq U_{n-1}^{n-1}$ by our induction assumption. Recalling that, by definition, $U_{n-1}^{R S W} \geq U_{n}^{R S W}-\psi_{n-1}\left(q_{n}^{R S W}\right)$, we thus have from $U_{n-1}^{R S W} \leq U_{n-1}^{n-1}$ that $\left(U_{n}^{R S W}, q_{n}^{R S W}\right)$ is admissible for problem $Z_{n}$. Since $\hat{U}_{n}^{n}$ is the optimal value for problem $Z_{n}$ we have therefore $U_{n}^{R S W} \leq \hat{U}_{n}^{n}$.

We show next that claim b) holds for type $n$. We notice first that $\left(\left(\hat{U}_{i}^{n}, \hat{q}_{i}^{n}\right)\right)_{1 \leq i \leq n}$ satisfies all assured claim constraints and all incentive constraints for Problem $X_{n}$ hold (by construction of $\left(\hat{U}_{n}^{n}, \hat{q}_{n}^{n}\right)$ and because $\left(\left(\hat{U}_{i}^{n}, \hat{q}_{i}^{n}\right)\right)_{1 \leq i \leq n-1}$ is a solution to $\left.X_{n-1}\right)$. The exante participation constraint from problem $X_{n-1}$ and the ex post participation constraint for $\left(\hat{U}_{n}^{n}, \hat{q}_{n}^{n}\right)$ from Problem $Z_{n}$ combined imply that $\left(\left(\hat{U}_{i}^{n}, \hat{q}_{i}^{n}\right)\right)_{1 \leq i \leq n-1}$ satisfies also the participation constraint of the Problem $X_{n}$. As in the proof of Proposition 2 (Appendix A, top of page 38) in Maskin and Tirole (1992), using that $W_{n-1}\left(q_{n-1}^{n-1}\right) \geq V_{n-1}=U_{n-1}^{n-1}$ by Lemma 3, one can show for the problem $Z_{n}$ that $\hat{q}_{n-1}^{n}<\hat{q}_{n}^{n}$. This, in turn, implies, given $\hat{q}_{i}^{n}=q_{i}^{n-1}$ for any $1 \leq i<n$, that all monotonicity constraints of the problem $X_{n}$ are also satisfied by the contract $\left(\left(\hat{U}_{i}^{n}, \hat{q}_{i}^{n}\right)\right)_{1 \leq i<n-1}$. Hence, the latter contract is admissible for $X_{n}$. Since $U_{n}^{n}=V_{n}$ is the optimal value for problem $X_{n}$ we have therefore $\hat{U}_{n}^{n} \leq U_{n}^{n}=V_{n}$. This completes the proof. 


\section{Proof of Theorem 5}

Assumption B is required in Theorem 5. We thus show next that Assumptioon B is not empty.

Lemma 7 A sufficient condition for Assumption $B$ is that

$$
\frac{W_{i}^{\prime}(q)}{\psi_{i-1}^{\prime}(q)}, \frac{f_{i-1}}{s_{i}} \text { and } \frac{f_{i-1}-f_{N-1}}{s_{i}} \text { are increasing in } i \text { for all } q \geq 0 \text {. }
$$

Proof. We first show that $\frac{f_{i-1}-\delta}{s_{i}}$ be strictly increasing in $i$ for any $\delta \in\left[0, f_{N-1}\right]$ for given $i, j$ with $j>i$. Suppose that $s_{j} \geq s_{i}$. We then have, by $\delta \geq 0$ and the assumed monotonicity of $\frac{f_{i-1}}{s_{i}}$, that $\frac{f_{j-1}-\delta}{s_{j}}-\frac{f_{i-1}-\delta}{s_{i}}>0$. Suppose now that $s_{j}<s_{i}$. We then have, by $\delta \leq f_{n-1}$ and the assumed monotonicity of $\frac{f_{i-1}-f_{N-1}}{s_{i}}$, that $\frac{f_{j-1}-\delta}{s_{j}}-\frac{f_{i-1}-\delta}{s_{i}}=\frac{f_{j-1}-f_{N-1}}{s_{j}}-$ $\frac{f_{i-1}-f_{N-1}}{s_{i}}+\left(f_{N-1}-\delta\right)\left(\frac{1}{s_{j}}-\frac{1}{s_{i}}\right)>0$.

By the first order conditions for a maximum, $q_{i}(\delta)$ is given by

$$
\frac{\delta-f_{i-1}}{s_{i}}=\frac{W_{i}^{\prime}(q)}{\psi_{i-1}^{\prime}(q)}
$$

for $q_{i}(\delta)>0$. Here the left-hand side is constant in $q$. Because $W_{i}$ is concave and $\psi_{i}$ convex, the quotient on the right-hand side is decreasing in $q$. As $i$ increases, the left-hand side shifts downward and the right-hand side upwards. $q_{i}(\delta)$ must hence increase.

To prove Theorem 5, we need a number of preliminary results.

We start with the following Lemma, which implies that the solution of $X_{n}(y)$ is the solution of $\tilde{X}_{n}(y)$, where, recall, the monotonicity constraints of $X_{n}(y)$ are ignored.

Lemma 8 Under Assumption B, the monotonicity constraints in problem $X_{n}(y)$ are slack (i.e. they could be ignored).

Proof. The proof is by induction on $n$. For $n=1$ there is nothing to show. Suppose the claim holds for all $1 \leq i<n$. Let $\left(\tilde{U}_{i}^{n}(y), \tilde{q}_{i}^{n}(y)\right)_{1 \leq i \leq n}$ be the solution of the optimization problem $\tilde{X}_{n}(y)$ with associated Lagrange multipliers $\left(\tilde{\sigma}_{i}, \tilde{\mu}_{i}\right)$. Let $\left(U_{i}^{n}(y), q_{i}^{n}(y)\right)_{1 \leq i \leq n}$ be the solution of $X_{n}(y)$ with associated Lagrange multipliers $\left(\sigma_{i}, \mu_{i}, \rho_{i}\right)$. Clearly, the first-order and complementarity conditions of the problem $X_{n}(y)$ with $\rho_{i}=0$ for all 
$0 \leq i \leq n$ are identical to the first-order and complementarity conditions of the problem $\tilde{X}_{n}(y)$. Moreover, it is easy to see that the multipliers $\left(\tilde{\sigma}_{i}, \tilde{\mu}_{i}\right)_{1 \leq i \leq n}$ and the solution $\left(\tilde{U}_{i}^{n}(y), \tilde{q}_{i}^{n}(y)\right)_{1 \leq i \leq n}$ satisfy the first-order conditions, the complementarity conditions associated with the incentive-compatibility and assured claim constraints, and the participation constraint of the problem $X_{n}(y)$, as long as $\rho_{i}=0$ for all $i$. As we show next, $\left(\tilde{q}_{i}^{n}(y)\right)_{1 \leq i \leq n}$ satisfy also the monotonicity constraints of problem $X_{n}(y)$.

Suppose, first, that none of the assured claim constraints $\mathrm{AC}_{i}, 1 \leq i<n$, are binding for $\left(\tilde{U}_{i}^{n}(y), \tilde{q}_{i}^{n}(y)\right)_{1 \leq i \leq n}$. Then $\tilde{g}_{i}^{n}(y)=\sum_{j=1}^{i} \tilde{\sigma}_{j}(y)=0$ for all $1 \leq i \leq n-1$ and hence $\tilde{\mu}_{i}=f_{i}$ by equation (12). By the first-order condition (11) we have $\tilde{q}_{i}^{n}(y)=q_{i}(0)$ with $q_{i}(\delta)$ as defined prior to Assumption B. By Assumption B we have $q_{1}(0) \leq q_{2}(0) \leq$ $\cdots \leq q_{n}(0)$. Secondly, suppose that some assured claim constraint is binding. In this case, there exists a largest $1 \leq k<n$ for which $\mathrm{AC}_{k}$ is binding. As stated in Remark 5 , Lemma 4 is valid. Thus, $\left(\tilde{q}_{i}^{n}(y)\right)_{1 \leq i \leq k}$ is part of the solution for problem $\tilde{X}_{k}(0)$ and hence $\tilde{q}_{1}^{n}(y) \leq \tilde{q}_{2}^{n}(y) \leq \cdots \leq \tilde{q}_{k}^{n}(y)$ by the induction assumption. By definition of $k$, none of the assured claim constraints $\mathrm{AC}_{i}, k+1 \leq i<n$, are binding, and hence (from 10) $\tilde{\sigma}_{i}=0$ for $k+1 \leq i<n$. Thus, $\tilde{g}_{i}^{n}(y)=\tilde{g}_{k}^{n}(y), \tilde{\mu}_{i}=f_{i}-\tilde{g}_{k}^{n}(y)$. This implies, by the first-order condition corresponding to (11) for $\tilde{X}_{n}(y)$, that $\tilde{q}_{i}^{n}(y)=q_{i}\left(g_{k}^{n}(y)\right)$ for all $k+1 \leq i \leq n-1$. Together with Assumption B and Lemma 4, the latter and the definition of $k$ imply that $\tilde{q}_{k}^{n}(y)<q_{k+1}\left(g_{k}^{n}(y)\right) \leq q_{k+2}\left(g_{k}^{n}(y)\right) \leq \cdots \leq q_{n}\left(g_{k}^{n}(y)\right)$. Accordingly, in both cases, we obtain that $\left(\tilde{g}_{i}^{n}(y)\right)_{1 \leq i \leq n}$ satisfies all the monotonicity constraints. Overall, the solution to $\tilde{X}_{n}(y)$ is hence, by uniqueness (Theorem 1), the solution to $X_{n}(y)$. This completes the proof.

Our last Lemma is:

Lemma 9 Suppose that $\left(U_{i}^{n}(y), q_{i}^{n}(y)\right)_{1 \leq i \leq n}$ is the solution to the optimization problem $\tilde{X}_{n}(y)$. Then

$$
\sum_{i=1}^{k} s_{i}\left(W_{i}\left(q_{i}^{n}(y)\right)-U_{i}^{n}(y)\right) \leq 0
$$

for any $k<n$ and the restricted solution $\left(U_{i}^{n}(y), q_{i}^{n}(y)\right)_{1 \leq i \leq k}$ is the solution to $\tilde{X}_{k}\left(y^{\prime}\right)$, where

$$
y^{\prime} \equiv-\sum_{i=1}^{k} \frac{s_{i}}{s_{k}}\left(W_{i}\left(q_{i}^{n}(y)\right)-U_{i}^{n}(y)\right)
$$


Proof. The inequality follows as in the first part of the proof of Lemma 4

Moving to the proof of the second part of the Lemma, note first that $\left(U_{i}^{n}(y), q_{i}^{n}(y)\right)_{1 \leq i \leq k}$ is admissible for $\tilde{X}_{k}\left(y^{\prime}\right)$. Suppose that $\left(U_{i}^{n}(y), q_{i}^{n}(y)\right)_{1 \leq i \leq k}$ is not a solution to $\tilde{X}_{k}\left(y^{\prime}\right)$. There is, then, a solution to $\tilde{X}_{k}\left(y^{\prime}\right)$ where type $k$ 's utility $\check{U}_{k}^{k}$ is higher than $U_{k}^{n}(y)$. Therefore,

$$
\check{U}_{k}^{k}>U_{k}^{n}(y) \geq U_{k+1}^{n}(y)-\psi_{k}\left(q_{k+1}^{n}(y)\right)
$$

By the maximum theorem we can thus find a solution to the problem $\tilde{X}_{k}\left(y^{\prime}-\varepsilon\right)$ with $\varepsilon>0$, denoted by $\left(\left(U_{i}^{k}\left(y^{\prime}-\varepsilon\right), q_{i}^{k}\left(\left(y^{\prime}-\varepsilon\right)\right)\right)\right)_{1 \leq i \leq k}$, such that $U_{k}^{k}\left(y^{\prime}-\varepsilon\right)>U_{k}^{n}(y)$ for sufficiently small $\varepsilon$. Clearly, $U_{k}^{k}\left(y^{\prime}-\varepsilon\right) \geq U_{k+1}^{n}(y)-\psi_{k}\left(q_{k+1}^{n}(y)\right)$. The new contract defined by

$$
\left(\hat{U}_{i}^{n}, \hat{q}_{i}^{n}\right)=\left\{\begin{array}{cc}
\left(U_{i}^{k}\left(y^{\prime}-\varepsilon\right), q_{i}^{k}\left(y^{\prime}-\varepsilon\right)\right) & \text { for } i \leq k \\
\left(U_{i}^{n}(y), q_{i}^{n}(y)\right) & \text { for } i>k
\end{array}\right.
$$

is thus admissible for problem $\tilde{X}_{n}(y)$ and has a slack participation constraint. The former is by construction, while the latter follows directly from the definitions of $y^{\prime}$ and $\left(U_{i}^{n}(y), q_{i}^{n}(y)\right)_{1 \leq i \leq N}$ and $\left(U_{i}^{k}\left(y^{\prime}-\varepsilon\right), q_{i}^{k}\left(y^{\prime}-\varepsilon\right)\right)_{1 \leq i \leq N}$. The new contract is also optimal since it gives, by construction, utility $U_{n}^{n}(y)$ to type $n$. This and the slackness of the participation constraint, under the new contract, of the problem $\tilde{X}_{n}(y)$ contradict Lemma 2.

We also need Theorem 7 in Myerson (1983), which we state for convenience (in terms of our set up) next. First, note that an undominated mechanism for weights $\left(\tau_{i}\right)_{1 \leq i \leq N}$ is a feasible mechanism that maximizes $\sum_{i} \tau_{i} U_{i}$. Let with some abuse of notation $\psi_{i j}(q)=$ $C_{i}(q)-C_{j}(q)$; that is, $\psi_{i}(q)=\psi_{i, i+1}(q)$. We have:

Proposition 4 (Myerson) A feasible mechanism $\left(U_{i}, q_{i}\right)_{1 \leq i \leq N}$ is a neutral optimum if and only if there exist sequences $\left.\left\{\left\{\tau_{i}^{\nu}\right\}_{i=1}^{N},\left\{\mu_{i i^{\prime}}^{\nu}\right\}_{i, i^{\prime}=1}^{N}, \gamma^{\nu},\left\{\omega_{i}^{\nu}\right\}_{i=1}^{N}\right\}\right\}_{\nu=1}^{\infty}$, with $\tau_{i}^{\nu}>0, \mu_{i i^{\prime}}^{\nu} \geq$ $0, \gamma^{\nu} \geq 0, \omega_{i}^{\nu}$ scalars, such that

$$
\left(\tau_{i}^{\nu}+\sum_{j} \mu_{i j}^{\nu}\right) \omega_{i}^{\nu}-\sum_{j} \mu_{j i}^{\nu} \omega_{j}^{\nu}=\gamma^{\nu} s_{i} V S_{i}^{\nu} \equiv\left(\gamma^{\nu} s_{i} W_{i}\left(q_{i}^{\nu}\right)-\sum_{j} \mu_{j i}^{\nu} \psi_{j i}\left(q_{i}^{\nu}\right)\right)
$$


and

$$
\lim _{\nu \rightarrow \infty} \sup \omega_{i}^{\nu} \leq U_{i}
$$

where $q_{i}^{\nu}, \mu_{i j}^{\nu}, 1 \leq i, j \leq N$, and $\gamma^{\nu}$ are evaluated at the optimal solution of the problem that determines the undominated allocation for given weights $\tau_{i}^{\nu}, 1 \leq i \leq N$.

Equation (18) corresponds to equation (8.8) in Myerson (1983). To interpret this theorem, Myerson refers to $\left(\omega_{i}^{\nu}\right)_{1 \leq i \leq N}$ as the warranted claim allocation (for a given $\nu$ ), and notes that the warranted claim allocation is the utility profile of a strong solution in an extension of the given model that has more mechanisms available than the original model. Thus, Theorem 7 in Myerson (1983) states, in effect, that neutral optimum is an undominated mechanism that dominates the limit of a sequence of abstract mechanisms that are strong solutions in an extended model which has more mechanisms available than the original model.

We are now ready to prove Theorem 5:

Proof of Theorem 5. We assume that Assumption B holds. Hence, by Lemma 8, any solution to a problem $X_{n}(y)$ for any $y$ features output $q_{i}^{n}(y)$ which is nondecreasing in $i$, and so we can ignore the monotonicity constraints $q_{i} \leq q_{i+1}$ in the following. Thus, we can assume that all multipliers $\rho_{i}$ are zero, and only the multipliers $\sigma_{i}$ and $\mu_{i}$ appear in the Lagrangian for the derivation of the assured allocation.

The virtual surplus associated with this solution is

$$
V S_{i}^{n}(y)=\frac{1}{s_{i}}\left[\mu_{i-1}^{n}(y) \psi_{i-1}^{n}\left(q_{i}^{n}(y)\right)+s_{i} W_{i}\left(q_{i}^{n}(y)\right)\right]
$$

Corresponding to equation (18) we can define the warranted claims $\omega_{i}^{n}(y)$ for problem $X_{n}(y)$ inductively over $i$ by

$$
\left(\sigma_{i}^{n}(y)+\mu_{i}^{n}(y)\right) \omega_{i}^{n}(y)-\mu_{i-1}^{n}(y) \omega_{i-1}^{n}(y)=s_{i} V S_{i}^{n}(y)
$$

Notice that the $\omega_{i}^{n}(y)$ are uniquely determined by Theorem 1. A solution to the problem $X_{n}(y)$ can now overall be described by $\left(U_{i}^{n}(y), q_{i}^{n}(y), \sigma_{i}^{n}(y), \mu_{i}^{n}(y)\right)_{1 \leq i \leq n}$ and has the assured claims $\left(\omega_{i}^{n}(y)\right)_{1 \leq i \leq n}$ associated with it.

We prove next the following claims for all $l \leq n$ and all $y \geq 0$ by induction on $n$ : 
1. $\omega_{i}^{l}(y) \leq U_{i}^{l}(y) \quad$ for $1 \leq i \leq l$.

2. For all $1 \leq i \leq l$ we have $\omega_{l}^{l}(0)=V_{l}$. Moreover, $\omega_{i}^{l}(y)=V_{i}$ for all $y \geq 0$ whenever $\sigma_{i}^{l}(y)>0$.

3. A solution to $X_{l}(0)$ is a neutral optimum for the restricted type set $\{1, \cdots, l\}$.

Note that all these claims are true for any $y \geq 0$ when $n=l=1$ : the solution to $X_{1}(0)$ is the first best, hence a strong solution and therefore a neutral optimum according to Myerson (1983). Therefore (3) holds. Since $\omega_{1}^{1}(y)=W_{1}\left(q_{1}^{1}(y)\right) \leq W_{1}\left(q_{1}^{1}(y)\right)+y=U_{1}^{1}(y)$ also (1) and the first part of (2) hold, while the second part is vacuously true.

Let $n>1$ and suppose that all three claims hold for all $l<n$ and $y \geq 0$. We prove them now for $l=n$ and $y=0$.

By Lemma 9 the solution to $X_{n}(0)$ induces the solution $X_{n-1}(y)$ with $y$ determined by $s_{n-1} y=-\sum_{i=1}^{n-1} s_{i}\left(W_{i}\left(q_{i}^{n}(0)\right)-U_{i}^{n}(0)\right) \geq 0$. We thus have $\omega_{i}^{n}(0)=\omega_{i}^{n-1}(y) \leq$ $U_{i}^{n-1}(y)=U_{i}^{n}(0)$ for all $i<n$, after using also claim (1) of the induction assumption and because we can choose the same Lagrange multipliers in both problems. This proves claim (1) for $y=0$ and all $i<l=n$. We also have $\sigma_{i}^{n}(0)=\sigma_{i}^{n-1}(y)$. Hence, we have by a similar argument $\omega_{i}^{n}(0)=\omega_{i}^{n-1}(y)=V_{i}$ for all $1 \leq i<n-1$ which satisfy $\sigma_{i}^{n-1}(y)>0$ by claim (2) of our induction assumption. If $\sigma_{n-1}^{n}(0)>0$, then necessarily $y=0$ by Lemmas 2 and 4 and hence $\omega_{n-1}^{n}(0)=\omega_{n-1}^{n-1}(0)=V_{n-1}$ again by the induction assumption. This proves the second part of claim (2) for $y=0$ and $l=n$. Per construction and from the definition of assured claims for $y=0$,

$$
\sum_{i=1}^{n-1} \sigma_{i}^{n}(0) \omega_{i}^{n}(0)+\left(\sigma_{n}^{n}(0)+\mu_{n}^{n}(0)\right) \omega_{n}^{n}(0)=\sum_{i=1}^{n} s_{i} V S_{i}^{n}(0)
$$

Equating the right-hand sides in (7) and (8), which both describe the Lagrangian we obtain for $y=0$

$$
\left(\sigma_{n}^{n}(0)+\mu_{n}^{n}(0)\right) U_{n}^{n}(0)=-\sum_{i=1}^{n-1} \sigma_{i}^{n}(0) V_{i}+\sum_{i=1}^{n} s_{i} V S_{i}^{n}(0)
$$

Above we use the complementarity conditions for an optimum to simplify the right-hand side of $(7)$ and we use the first order condition (10) to simplify (8), recalling that all $\rho_{i}$ 
are zero. Since $V_{n}=U_{n}^{n}(0)$ by definition we obtain from (21) and (22)

$$
\sum_{i=1}^{n-1} \sigma_{i}^{n}(0) \omega_{i}^{n}(0)+\left(\sigma_{n}^{n}(0)+\mu_{n}^{n}(0)\right) \omega_{n}^{n}(0)=\sum_{i=1}^{n-1} \sigma_{i}^{n}(0) V_{i}+\left(\sigma_{n}^{n}(0)+\mu_{n}^{n}(0)\right) V_{n}
$$

We conclude, after using the second part of claim (2) for $y=0$ and $i<n$, overall that $\omega_{n}^{n}(0)=V_{n}=U_{n}^{n}(0)$. This proves claims (1) and the first part of (2) for $y=0$ and $i=l=n$.

Next, we construct a sequence $\left(U_{i}^{n}\left(0, \varepsilon_{\nu}\right), q_{i}^{n}\left(0, \varepsilon_{\nu}\right), \sigma_{i}^{n}\left(0, \varepsilon_{\nu}\right), \mu_{i}^{n}\left(0, \varepsilon_{\nu}\right), \omega_{i}^{n}\left(0, \varepsilon_{\nu}\right)\right)_{1 \leq i \leq n}$ for $\varepsilon_{\nu}>0, \lim _{\nu \rightarrow \infty} \varepsilon_{\nu}=0$ which converges to $\left(U_{i}^{n}(0), q_{i}^{n}(0), \sigma_{i}^{n}(0), \mu_{i}^{n}(0), \omega_{i}^{n}(0)\right)_{1 \leq i \leq n}$ such that the first-order conditions for the Lagrangian and the complementarity conditions always hold, where the $\omega_{i}^{n}\left(0, \varepsilon_{\nu}\right)$ are defined as above with respect to the "virtual surpluses" $V S_{i}^{\varepsilon_{\nu}}$ and where $\sigma_{i}^{n}\left(0, \varepsilon_{\nu}\right), \mu_{i}^{n}\left(0, \varepsilon_{\nu}\right) \geq \varepsilon_{\nu}$. Namely, set $\varepsilon_{\nu}=1 / \nu$ for any integer $\nu$ sufficiently large and set $\sigma_{i}^{n}\left(0, \varepsilon_{\nu}\right)=\sigma_{i}^{n}(0)$ if $\sigma_{i}^{n}(0)>0$ and $\sigma_{i}^{n}\left(0, \varepsilon_{\nu}\right)=\varepsilon_{\nu}$ otherwise. Set $g_{i}^{\varepsilon_{\nu}}=\sum_{j=1}^{i} \sigma_{j}^{n}\left(0, \varepsilon_{\nu}\right)$. Then $\mu_{i}^{n}\left(0, \varepsilon_{\nu}\right)=f_{i}-g_{i}^{\varepsilon_{\nu}}$ is non-negative for sufficiently large $\nu$. The $q_{i}^{n}\left(0, \varepsilon_{\nu}\right)$ are then uniquely determined by $\mu_{i-1}^{n}\left(0, \varepsilon_{\nu}\right)$ from the condition (11) using $\rho_{i}=\rho_{i-1}=0$. The "virtual surpluses" $V S_{i}^{\varepsilon_{\nu}}$ are then derived from the formula (19) and the "warranted claims" $\omega_{i}^{n}\left(0, \varepsilon_{\nu}\right)$ from (20). Continuity and the uniqueness of the solution implies as $\nu \rightarrow \infty$ that $\omega_{i}^{n}\left(0, \varepsilon_{\nu}\right) \rightarrow \omega_{i}^{n}(0), \mu_{i-1}^{n}\left(0, \varepsilon_{\nu}\right) \rightarrow \mu_{i-1}^{n}(0)$ etc. The $\sigma_{i}^{n}\left(0, \varepsilon_{\nu}\right), \sigma_{i}^{n}(0)$ and $\omega_{i}^{n}(0)$ etc. play hereby the role of the utility weights $\tau_{i}^{\nu}$, $\lim _{\nu \rightarrow \infty} \tau_{i}^{\nu}$, and the warranted allocation $\omega_{i}$, etc. in the characterization of neutral optima in Proposition 4 when the type space is restricted to the types $i=1, \cdots, n$ and the prior for each type is $s_{i} / f_{i}$. Thus, from claim (1) with $y=0$ the $n$-assured allocation is a neutral optimum by Theorem 7 in Myerson (1983) and so claim (3) holds for $l=n$.

It remains to show claim (1) and the second part of claim (2) for $l=n$ and $y>0$.

Suppose that $\sigma_{i}^{n}(0)=0$ for any $i<n$ in the solution to $X_{n}(0)$. It is immediately seen that

$$
\left(U_{i}^{n}(0)+\frac{s_{n} y}{n}, q_{i}^{n}(0), 0, \mu_{i}^{n}(0)\right)_{1 \leq i \leq n}
$$

is a solution to the problem $X_{n}(y)$ for all $y>0$ because $U_{i}^{n}(y)=U_{i}^{n}(0)+\frac{s_{n} y}{n}>U_{i}^{n}(0) \geq$ $V_{i}$ (and hence $\sigma_{i}^{n}(y)=0$ ) for all $i \leq n$ and $\left(q_{i}^{n}(0), \mu_{i}^{n}(0)\right)_{1 \leq i \leq n}$ satisfy the first-order conditions of the problem $X_{n}(y)$. Clearly, then, the second part of claim (2) is trivially 
satisfied and, furthermore, $\omega_{i}^{n}(y)=\omega_{i}^{n}(0)$. Thus, given $\omega_{i}^{n}(0) \leq U_{i}^{n}(0)$ by claim (1) for $y=0$, we have $U_{i}^{n}(y)>\omega_{i}^{n}(y)$ for any $y>0$ and $i \leq n$.

Suppose next that there is some $i<n$ such that $\sigma_{i}^{n}(0)>0$ in the solution to $X_{n}(0)$ and let $k<n$ be the largest such index. By continuity, as we increase $y$ we will have $\sigma_{k}^{n}(y)>0$ in some maximal interval $0 \leq y<\bar{y}$, which can easily be shown to be of finite length. In this interval we will first show that all $U_{i}^{n}(y)$ for $i>k$ are strictly increasing in $y$. Hence the assured claim constraints $U_{i}^{n}(y) \geq V_{i}$ cannot become binding for $k<i<n$. At $\bar{y}$ the largest index $k^{\prime}$ for which $\sigma_{k^{\prime}}^{n}(\bar{y})>0$ is thus necessarily smaller than $k$. We will also show that claim (1) and the second part of claim (2) hold for all $0 \leq y<\bar{y}$. One can now apply exactly the same arguments on the maximal interval $\bar{y} \leq y<\bar{y}^{\prime}$ where $\sigma_{k^{\prime}}(y)>0$ as on the interval $0 \leq y<\bar{y}$.

Proceeding by induction in this way one will eventually arrive at a level of $\tilde{y}$ from which onwards all $\sigma_{i}^{n}(y), i<n$, are zero. From there onwards a further increase in $y$ does not affect the multipliers $\mu_{i}^{n}(y), i<n$, anymore, which are now at their maximal value $\mu_{i}^{n}(y)=f_{i}$. Hence, neither $q_{i}^{n}(y)$ nor the virtual surpluses nor $\omega_{i}^{n}(y)$ change as $y$ increases. Only the $U_{i}^{n}(y)$ are increased, all in the same way because all incentive constraints $\mathrm{IC}_{i}$ are binding (recall Lemma 2). Thus, once all $\sigma_{i}^{n}(y)$ are zero for $i<n$ they remain so for all $y^{\prime} \geq y$, and if $\omega_{i}^{n}(y) \leq U_{i}^{n}(y)$ holds in addition, this remains so for all $y^{\prime} \geq y$. Hence, claim (1) and the second part of (2) hold for all $y \geq \bar{y}$ if they hold for $y<\bar{y}$.

To complete, given this outline, the proof, we now show (a) that $U_{i}^{n}(y)$, for $i>k$, are strictly increasing in $y$, and (b) that claim (1) and the second part of (2) hold for all $0 \leq y<\bar{y}$

Since $U_{k}^{n}(y)=V_{k}$ in the solution to $X_{n}(y)$, for all $0 \leq y<\bar{y}$, the solution in question induces by Lemma 4 the solution to $X_{k}(0)$, and so we have $\omega_{i}^{n}(y)=\omega_{i}^{k}(0) \leq U_{i}^{k}(0)=$ $U_{i}^{n}(y)$ for all $0 \leq y<\bar{y}$ and $i \leq k$. Moreover, $U_{i}^{n}(y)=U_{i}^{k}(0)=V_{i}=\omega_{i}^{k}(0)=\omega_{i}^{n}(y)$ for all $i \leq k$ which satisfy $\sigma_{i}^{k}(0)=\sigma_{i}^{n}(y)>0$, in particular for $i=k$. Thus, claim (1) and the second part of (2) are proved in the interval for all $i \leq k$. None of these variables change as we vary $y$ in the interval. Since, by Lemma $4, \mu_{l}^{n}(y)=f_{l}-g_{k}^{n}(y)=f_{l}-g_{k-1}^{n}(0)-\sigma_{k}^{n}(y)$, a marginal change in $y$ affects $\sigma_{k}^{n}(y)$ and thereby affects all $\mu_{l}^{n}(y), l \geq k$, in the same way, 
i.e.

$$
\frac{d \mu_{l}^{n}}{d y}=\frac{d \mu_{j}^{n}}{d y}=-\frac{d \sigma_{k}^{n}}{d y} \text { for all } l, j \geq k
$$

We abbreviate $\frac{d \mu}{d y}=\frac{d \mu_{l}^{n}}{d y}$ for $l \geq k$. All incentive constraints $\mathrm{IC}_{l}$ must be binding for $l>k$ by Lemma 2 . Therefore, $U_{l}^{n}(y)=U_{k}^{n}(y)+\sum_{i=k+1}^{l} \psi_{i-1}\left(q_{i}^{n}(y)\right)$. When we now slightly increase $y$ we obtain

$$
\frac{d U_{l}^{n}(y)}{d y}=\sum_{i=k+1}^{l} \psi_{i-1}^{\prime}\left(q_{i}^{n}(y)\right) \frac{d q_{i}^{n}(y)}{d y}
$$

The first-order condition for $q_{i}^{n}(y)$ is $\mu_{i-1}^{n}(y) \psi_{i-1}^{\prime}\left(q_{i}^{n}(y)\right)+s_{i} W_{i}^{\prime}\left(q_{i}^{n}(y)\right)=0$. Differentiation yields

$$
\begin{aligned}
& \frac{d \mu}{d y} \psi_{i-1}^{\prime}\left(q_{i}^{n}(y)\right)+\left[\mu_{i-1}^{n}(y) \psi_{i-1}^{\prime \prime}\left(q_{i}^{n}(y)\right)+s_{i} W_{i}^{\prime \prime}\left(q_{i}^{n}(y)\right)\right] \frac{d q_{i}^{n}(y)}{d y}=0 \\
& \frac{d q_{i}^{n}(y)}{d y}=-\frac{\psi_{i-1}^{\prime}\left(q_{i}^{n}(y)\right)}{\mu_{i-1}^{n}(y) \psi_{i-1}^{\prime \prime}\left(q_{i}^{n}(y)\right)+s_{i} W_{i}^{\prime \prime}\left(q_{i}^{n}(y)\right)} \frac{d \mu}{d y}
\end{aligned}
$$

which has the same sign as $d \mu / d y$ by the second-order conditions, and hence

$$
\frac{d U_{l}^{n}(y)}{d y}=\left(-\sum_{i=k+1}^{l} \frac{\left(\psi_{i-1}^{\prime}\left(q_{i}^{n}(y)\right)\right)^{2}}{\mu_{i-1}^{n}(y) \psi_{i-1}^{\prime \prime}\left(q_{i}^{n}(y)\right)+s_{i} W_{i}^{\prime \prime}\left(q_{i}^{n}(y)\right)}\right) \frac{d \mu}{d y}
$$

where the term in brackets is positive. For $l=n$ we obtain $\frac{d \mu}{d y}>0$ since an increase in $y$ slackens the participation constraint and hence $U_{n}^{n}(y)$ must increase. (Formally $\frac{d U_{n}^{n}(y)}{d y}>0$ follows by applying the envelope theorem to the Lagrangian.) We see, in turn, that all $U_{l}^{n}(y)$ are strictly increasing for all $0 \leq y<\bar{y}$ and $k<l<n$. Therefore, all assured claim constraints remain slack. In particular, $\sigma_{i}^{n}(y)=0$ for all $i>k$ and, hence, the second part of claim (2) is proved for all $0 \leq y<\bar{y}$ and $1 \leq i<l=n$.

We continue with the proof of claim (1). For $l>k$ we have for the assured claims ${ }^{34}$

$$
\mu_{l}^{n}(y) \omega_{l}^{n}(y)-\mu_{k}^{n}(y) \omega_{k}^{n}(y)=\sum_{i=k+1}^{l}\left[\mu_{i-1}^{n}(y) \psi_{i-1}\left(q_{i}^{n}(y)\right)+s_{i} W_{i}\left(q_{i}^{n}(y)\right)\right]
$$

\footnotetext{
${ }^{34}$ For this calculation it is convenient to set $\sigma_{n}^{n}(y)=0$.
} 
We obtain

$$
\begin{aligned}
\frac{d}{d y}\left[\mu_{l}^{n}(y) \omega_{l}^{n}(y)-\mu_{k}^{n}(y) \omega_{k}^{n}(y)\right] & =\frac{d \mu}{d y}\left(\omega_{l}^{n}(y)-\omega_{k}^{n}(y)\right)+\mu_{l}^{n}(y) \frac{d \omega_{l}^{n}}{d y} \\
& =\left[\sum_{i=k+1}^{l} \psi_{i-1}\left(q_{i}^{n}(y)\right)\right] \frac{d \mu}{d y}
\end{aligned}
$$

where the first and second term are equal because $\omega_{k}^{n}(y)=V_{k}$ is constant in $y$ while the first and the third term are equal by the product rule and by using the first order condition for $q_{i}^{n}(y)$ maximizing the virtual surplus. Therefore

$$
\frac{d \omega_{l}^{n}}{d y}=\left[\sum_{i=k+1}^{l} \psi_{i-1}\left(q_{i}^{n}(y)\right)+\omega_{k}^{n}(y)-\omega_{l}^{n}(y)\right] \frac{d \mu}{d y} / \mu_{l}^{n}(y)
$$

Since $\omega_{k}^{n}(y)=U_{k}^{n}(y)$, by the second part of claim (2) and because $U_{k}^{n}(y)=V_{k}$, and $U_{l}^{n}(y)=U_{k}^{n}(y)+\sum_{i=k+1}^{l} \psi_{i-1}\left(q_{i}^{n}(y)\right)$ by the incentive constraints, it follows that

$$
\frac{d \omega_{l}^{n}}{d y}=\left(U_{l}^{n}(y)-\omega_{l}^{n}(y)\right) \frac{d \mu}{d y} / \mu_{l}^{n}(y)
$$

The proof is now concluded by showing the following statement to be true.

Consider the maximal interval $[0, \bar{y})$ of all values of $y$ for which $\sigma_{k}^{n}(y)>0$. Then $\omega_{l}^{n}(y) \leq U_{l}^{n}(y)$ for all $l>k$ and for all $y$ in this interval.

The proof of the statement is by contradiction. Suppose $U_{l}^{n}(y)<\omega_{l}^{n}(y)$ for some $0 \leq y<\bar{y}$. Let $\hat{y}=\inf \left\{y \mid U_{l}^{n}(y)<\omega_{l}^{n}(y)\right\}$. For $y=\hat{y}$ we have $U_{l}^{n}(y)=\omega_{l}^{n}(y)$ because both functions are continuous and $U_{l}^{n}(0) \geq \omega_{l}^{n}(0)$. Hence $\frac{d \omega_{l}^{n}}{d y}{ }_{\mid y=\hat{y}}=0$. Since $\frac{d U_{l}^{n}}{d y}>0$ we have $\frac{d\left(U_{l}^{n}-\omega_{l}^{n}\right)}{d y} \mid y=\hat{y}>0$. It follows that $U_{l}^{n}(y)>\omega_{l}^{n}(y)$ for all small $y>\hat{y}$, in contradiction to the definition of $\hat{y}$. 